\title{
On Standardized Model Integration Automated Validation in Aircraft Systems Simulation
}

\section{Robert Hällqvist}





\section{On Standardized Model Integration}

Automated Validation in Aircraft System Simulation

\section{Robert Hällqvist}

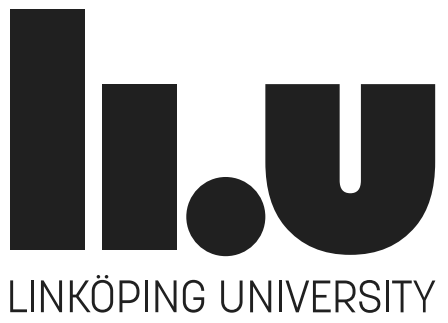

Division of Fluid and Mechatronic Systems Department of Management and Engineering

Linköping University, SE-581 83 Linköping, Sweden 
Copyright (C) Robert Hällqvist, 2019

On Standardized Model Integration

Automated Validation in Aircraft System Simulation

ISBN 978-91-7929-929-3

ISSN 0280-7971

Cover: Robert Hällqvist, 2019

\section{Distributed by:}

Division of Fluid and Mechatronic Systems

Department of Management and Engineering

Linköping University

SE-581 83 Linköping, Sweden

Printed in Sweden by LiU-Tryck, Linköping 2019. 
To Carolina, My, and Emmett 



\section{Abstract}

Designing modern aircraft is not an easy task. Today, it is not enough to optimize aircraft sub-systems at a sub-system level. Instead, a holistic approach is taken whereby the constituent sub-systems need to be designed for the best joint performance. The State-of-the-Art (SotA) in simulating and exchanging simulation models is moving forward at a fast pace. As such, the feasible use of simulation models has increased and additional benefits can be exploited, such as analysing coupled sub-systems in simulators. Furthermore, if aircraft sub-system simulation models are to be utilized to their fullest extent, opensource tooling and the use of open standards, interoperability between domain specific modeling tools, alongside robust and automated processes for model Verification and Validation $(\mathrm{V} \& \mathrm{~V})$ are required.

The financial and safety related risks associated with aircraft development and operation require well founded design and operational decisions. If those decisions are to be founded upon information provided by models and simulators, then the credibility of that information needs to be assessed and communicated. Today, the large number of sensors available in modern aircraft enable model validation and credibility assessment on a different scale than what has been possible up to this point. This thesis aims to identify and address challenges to allow for automated, independent, and objective methods of integrating sub-system models into simulators while assessing and conveying the constituent models aggregated credibility.

The results of the work include a proposed method for presenting the individual models' aggregated credibility in a simulator. As the communicated credibility of simulators here relies on the credibility of each included model, the assembly procedure itself cannot introduce unknown discrepancies with respect to the System of Interest (SoI). Available methods for the accurate simulation of coupled models are therefore exploited and tailored to the applications of aircraft development under consideration. Finally, a framework for automated model validation is outlined, supporting on-line simulator credibility assessment according to the presented proposed method. 


\section{Acknowledgments}

This work was carried out at the Division of Fluid and Mechatronic Systems (FLUMES) at the Department of Management and Engineering (IEI) at Linköping University. The research has been funded by VINNOVA and Saab Aeronautics via the two research projects Model Validation - from Concept to Product and Open Cyber-Physical System Model-Driven Certified Development (OpenCPS).

I would first and foremost like to express my gratitude to my supervisor Professor Petter Krus. Thank you Petter for your guidance, devotion, and for being an inspiration. I would also like to sincerely thank my co-supervisors Dr. Magnus Eek and Dr. Robert Braun. Thank you for your great dedication to the addressed topics, your guidance, and being admirable individuals. Many thanks to all of the OpenCPS project members, you all made the project a most pleasant experience. I would particularly like to thank Dr. Lenart Ochel and Sebastien Revol for their extensive efforts in tool development. I would like to acknowledge my former and current line managers Dr. Hampus Gavel and Peter Gotenstam. Thank you both for supporting me in pursuing my lifelong dream to work with research and for fending off industrial assignments in favour of my academic studies. I would also like to thank all of my colleagues at the department of Systems Simulation and Concept Design. Thank you all for your support and simply for being good friends. Many thanks to all of my colleagues at FLUMES. Thank you for the interesting talks about everything under the sun. I felt welcome the minute I set foot in the university. To my parents Torbjörn and Gullvi. Thank you for your unconditional interest in all of my endeavours, thank you for being great parents, and thank you for being wonderful grandparents. Finally, thank you Carolina for being the wonderful person and fiancée that you are. Thank you for your patience and love; until death do us part. Thank you My and Emmett for being the two most wonderful children in the world, making sure that both I and Carolina stay on our toes.

Linköping, November 2019

Robert Hällqvist 


\section{Abbreviations}

A/C Aircraft

$\mathrm{AE} \quad$ Algebraic Equation

CAS Credibility Assessment Scale

CFD Computational Fluid Dynamics

CI Configuration Item

CM Configuration Management

CS Co-Simulation

DAE Differential Algebraic Equation

DE Differential Equation

DoV Domain of Validation

DSM Design Structure Matrix

ECS Environmental Control System

FEM Finite Element Methods

FFT Fast Fourier Transform

FMI Functional Mock-up Interface

FMU Functional Mock-up Unit

GUI Graphical User Interface

$\mathrm{H} / \mathrm{W} \quad$ Hardware

HIL Hardware In the Loop

HLA High Level Architecture

INCOSE International Council on Systems Engineering

IP Intellectual Property

IV\&V Independent Verification and Validation

M\&S Modeling and Simulation

MA Modelica Association

MBSE Model Based System Engineering

ME Model Exchange

MSL Modelica Standard Library

MST Master Simulation Tool

NASA National Aeronautics and Space Administration

NFFP Nationellt Flygtekniskt Forskningsprogram

OD Operational Domain 


$\begin{array}{ll}\text { OpenCPS } & \text { Open Cyber-Physical System Model-Driven } \\ & \text { Certified Development } \\ \text { PCMM } & \text { Predictive Capability Maturity Model } \\ \text { PDE } & \text { Partial Differential Equation } \\ \text { PEM } & \text { Prediction Error Method } \\ \text { S/W } & \text { Software } \\ \text { SME } & \text { Subject Matter Expert } \\ \text { SoI } & \text { System of Interest } \\ \text { SoS } & \text { System-of-Systems } \\ \text { SotA } & \text { State-of-the-Art } \\ \text { SRQ } & \text { System Response Quantity } \\ \text { SSD } & \text { System Structure Description } \\ \text { SSP } & \text { System Structure and Parameterization } \\ \text { TLM } & \text { Transmission Line Method } \\ \text { TRL } & \text { Technology Readiness Level } \\ \text { UML } & \text { Unified Modeling Language } \\ \text { UQ } & \text { Uncertainty Quantification } \\ \text { V\&V } & \text { Verification and Validation } \\ \text { VPMM } & \text { Validation Process Maturity Model } \\ \text { VV\&A } & \text { Verification Validation and Accreditation } \\ \text { XML } & \text { Extensible Markup Language } \\ \text { xtUML } & \text { Executable UML }\end{array}$




\section{Nomenclature}

Area

$\left[\mathrm{m}^{2}\right]$

Speed of sound

Bulk modulus

Wave variable $i$

Distance from OD grid point $i$ to validation point

extrapolation penalty

Prediction error

Nearest neighbor coverage

System level validation metric accounting for coverage

Number of grid points in OD

Number of available samples

Angular frequency

Pressure

Pressure deviation from initial value $p\left(t_{0}\right)$

Phase shift

Volumetric flow

Net volumetric flow

Density

Time

Initial time

Optimal model parameter vector $[\mathrm{m} / \mathrm{s}]$

[Pa]

[Pa]

[-]

[-]

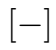

[-]

[-]

[-] $[\mathrm{rad} / \mathrm{s}]$

[Pa]

$[\mathrm{Pa}]$

$\left.{ }^{\circ}\right]$

$\left[\mathrm{m}^{3} / \mathrm{s}\right]$

$\left[\mathrm{m}^{3} / \mathrm{s}\right]$

$\left[\mathrm{kg} / \mathrm{m}^{3}\right]$

[s]

[s] 
Vector of model parameters

$T_{\text {macro }} \quad$ Time at simulator level

$[\mathrm{s}]$

$T_{s} \quad$ Sampling interval

$[\mathrm{S}]$

$\Delta t_{T L M} \quad$ Time delay of transmission line

V

Volume

$\left[\mathrm{m}^{3}\right]$

$v$

Velocity

$[\mathrm{m} / \mathrm{s}]$

$V_{E} \quad$ System level validation metric

$\left[\mathrm{m}^{3}\right]$

$Z_{c}$

Characteristic impedance

$\left[\mathrm{sPa} / \mathrm{m}^{3}\right]$ 


\section{Papers}

The listed papers constitute the basis of this compilation thesis. The papers are appended, chronologically, in the state in which they were originally published with only minor changes in format. The papers are referred to by their roman numerals throughout the thesis. In papers [II] and [IV] the first listed author is the main author. In papers [I] and [III], both authors are listed as main authors. Hällqvist contributed with planning, manuscript writing, implementation, and result evaluation in Paper [I]. In Paper [III], the author of this thesis contributed with modeling side functionality whereas Dr. Robert Braun was responsible for the tooling side conducted development.

[I] Magnus Eek, Robert Hällqvist, Hampus Gavel, and Johan Ölvander. "Development and Evaluation of a Concept for Credibility Assessment of Aircraft System Simulators". In: AIAA Journal of Aerospace Information Systems 13.6 (June 2016), pp. 219-233. DOI: https : //doi . org/10.2514/1. I010391.

[II] Robert Hällqvist, Magnus Eek, Robert Braun, and Petter Krus. "Methods for automating model validation: steady-state identification applied on gripen fighter environmental control system measurements". In: Proceedings of the 30th Congress of the International Council of the Aeronautical Sciences. DCC, Daejon, Korea: International Council of the Aeronautical Sciences, 2016. ISBN: 978-3-932182-85-3.

[III] Robert Braun, Robert Hällqvist, and Dag Fritzon. "TLM-based Asynchronous Co-simulation with the Functional Mockup Interface". In: $I U$ TAM Symposium on Solver Coupling and Co-Simulation. Darmstadt, Germany: Springer International Publishing, 2017. DoI: https://doi. org/10.1007/978-3-030-14883-6.

[IV] Robert Hällqvist, Jörg Schminder, Magnus Eek, Robert Braun, Roland Gårdhagen, and Petter Krus. "A Novel FMI and TLM-based Desktop Simulator for Detailed Studies of Thermal Pilot Comfort". In: Proceedings of the 31st Congress of the International Council of the Aeronautical Sciences. International Council of the Aeronautical Sciences, 2018. ISBN: 978-3-932182-88-4. 


\section{Other publications}

The publications listed below are not included in the thesis. However, they do constitute an important part of the work and its background. In Paper [VII], the first two listed authors have contributed equally to the presented work and are listed as main authors. In Paper [VIII], the thesis author contributed with testing, posing requirements, and implementing the aircraft sub-systems simulator.

[V] Robert Hällqvist, Magnus Eek, Ingela Lind, and Hampus Gavel. "Validation Techniques Applied on the Saab Gripen Fighter Environmental Control System Model". In: Proceedings of the 56th Conference on Simulation and Modelling (SIMS 56). Linköping, Sweden: Linköping University Electronic Press, Linköpings universitet, Nov. 25, 2015. ISBN: 978-91-7685-900-1. DOI: 10.3384/ecp15119.

[VI] Robert Hällqvist, Robert Braun, and Petter Krus. "Early Insights on FMI-based Co-Simulation of Aircraft Vehicle Systems". In: Proceedings of the 15th Scandinavian International Conference on Fluid Power. Linköping, Sweden: Linköping University Electronic Press, 2017. ISBN: 978-91-7685-369-6.

[VII] Jörg Schminder, Robert Hällqvist, Magnus Eek, and Roland Gårdhagen. "Pilot Performance and Heat Stress Assessment Support Using a Cockpit Thermoregulatory Simulation Model". In: Proceedings of the 31 st Congress of the International Council of the Aeronautical Sciences. International Council of the Aeronautical Sciences, 2018. ISBN: 978-3-932182-88-4.

[VIII] Lennart Ochel et al. "OMSimulator - Integrated FMI and TLM-based Co-simulation with Composite Model Editing and SSP". In: Proceeding of the 13th International Modelica Conference. Mar. 4, 2019. DOI: 10. 3384/ecp1915769. 


\section{Contents}

1 Introduction 1

1.1 Background . . . . . . . . . . . . . . . . . 1

1.2 Aim and Research Questions . . . . . . . . . . . . 3

1.3 Delimitations . . . . . . . . . . . . . . . . . . . . 4 4

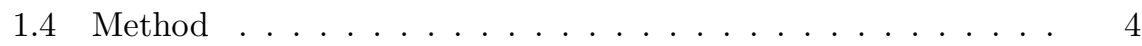

1.5 Overview of Appended Papers . . . . . . . . . . . . . 5

1.6 Thesis Outline . . . . . . . . . . . . . . . . 6

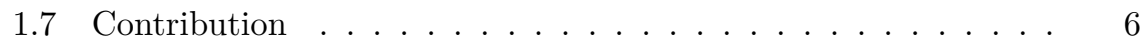

2 Simulator Applications in Aircraft Vehicle Systems Development 9

2.1 Concepts and Terminology . . . . . . . . . . . . . . . 10

2.2 Modeling and Simulation of Aircraft Sub-Systems . . . . . . . . 11

2.2 .1 Small-scale simulators . . . . . . . . . . . . . . . 12

2.2 .2 Mid-scale simulators . . . . . . . . . . . . . . . . 12

2.2 .3 Large-scale simulators . . . . . . . . . . . . . . . 13

2.2.4 Overview of simulator applications at Saab Aeronautics 14

2.3 Integrating Models into Simulators . . . . . . . . . . . . 16

3 Standards for Model Integration $\quad 19$

3.1 Exchange of Models . . . . . . . . . . . . . . . . . . 20

3.2 Simulator Composition and Exchange . . . . . . . . . . 21

3.3 Interoperability with FMI and SSP . . . . . . . . . . . 22

4 Co-Simulation of Coupled Sub-System Models 25

4.1 Challenges . . . . . . . . . . . . . . . . 26

4.2 Transmission Line Method (TLM) $\ldots \ldots \ldots \ldots$

4.3 Transmission Line Characteristics . . . . . . . . . . . . . . 30

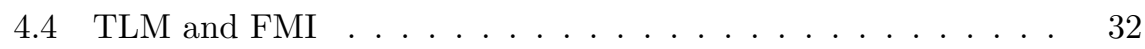

4.4.1 Accessing interpolation data . . . . . . . . . . . 33 
5 Credibility Assessment 39

5.1 Concepts and Terminology . . . . . . . . . . . . . . . 39

5.1 .1 Domains of model operation . . . . . . . . . . . . . 40

5.1 .2 Operational domain coverage . . . . . . . . . . . . 41

5.2 Automating Model Validation . . . . . . . . . . . . . . . . . 42

5.2 .1 Pre-processing . . . . . . . . . . . . . . . . . 43

5.2 .2 Post-processing . . . . . . . . . . . . . . . . 46

5.3 Automating Simulator Credibility Assessment . . . . . . . . 47

6 Discussion and Conclusions 49

6.1 Discussion . . . . . . . . . . . . . . . . . . . . . 49

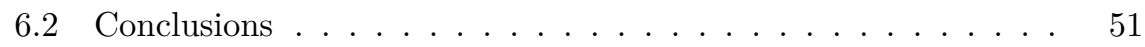

6.3 Outlook and Future Work . . . . . . . . . . . . . . . 53

A FMI and SSP Examples $\quad 55$

A.1 Exchange of Models . . . . . . . . . . . . . . . 55

A.2 Simulator Exchange $\ldots \ldots \ldots \ldots \ldots \ldots$

B Derivation of TLM Equations $\quad 61$

C Validation Experiments $\quad 65$

C.1 Experiment $2 \ldots \ldots \ldots \ldots \ldots$. . . . . . . . . . . . 66

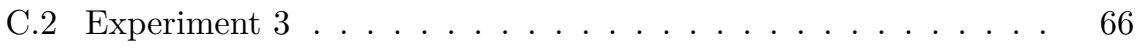

$\begin{array}{ll}\text { Bibliography } & 69\end{array}$

\section{Appended Papers}

I A Concept for Credibility Assessment of Aircraft System Simulators

II Methods for Automating Model Validation: Steady-State Identification Applied on Gripen Fighter Environmental Control System Measurements

III TLM-based Asynchronous Co-simulation with the Functional Mockup Interface

IV A Novel FMI and TLM-Based Desktop Simulator For Detailed Studies of Thermal Pilot Comfort 


\section{1 \\ Introduction}

Modeling and Simulation (M\&S) is a broad concept that is applied to varying extents at different companies and projects. Here, M\&S is considered to be the development and use of models that together describe selected behavior of a complete Aircraft (A/C) or A/C sub-system. Saab Aeronautics have been investing heavily for many years in the use of models during $\mathrm{A} / \mathrm{C}$ development in terms of M\&S methods and the available tooling and tool support. Currently, the need for $M \& S$ is clear. M\&S is vital in predicting the joint behavior of the ever-so-tightly coupled A/C sub-systems. A/C are being designed for decades of operation in widely different conditions. The corresponding models and simulators, see Definition 2.1.2 and Definition 2.1.1 respectively, need to be used throughout the entire A/C life-cycle. Sustainable methods for model and simulator development as well as Verification and Validation (V\&V) are therefore a necessity. The focus of this thesis is consequently on integrating simulation models into simulators. Integration here concerns the complete process of developing, accurately simulating, and assessing the credibility of simulator applications. The presented focus is a result of industrial experience indicating that the manual effort and know-how associated with the integration of models into simulators is time consuming and error prone. In addition, tool specific M\&S solutions tend to result in vendor-lock-in effects that limit the beneficial reuse of models [1]. Improving on the SotA in industry, incorporating open tools and standards, will therefore be of great benefit to the development and maintenance of $\mathrm{A} / \mathrm{C}$ sub-systems in general.

\subsection{Background}

Models are necessary in order to develop modern A/C. Today, the comprising $\mathrm{A} / \mathrm{C}$ sub-systems are closely interconnected enabling advanced controlling software optimization, for example, maximizing overall aircraft performance while providing a comfortable working environment for pilots. Designing an 
$\mathrm{A} / \mathrm{C}$ for nominal performance in the most demanding conditions is no longer a feasible approach when developing competitive fighter A/C. Instead, the system's dynamic properties need to be considered. This means that the use of simulation models is required. As models mirror carefully selected aspects of the real sub-system, from now on referred to as the System of Interest (SoI), the models tend to be complex and as a result time consuming to develop, maintain, and execute. In addition, sub-systems cannot be analyzed separately; instead, a holistic approach is needed. Detailed simulations of large portions of a complete $\mathrm{A} / \mathrm{C}$ are therefore pursued.

To fully retain the value of investments in $\mathrm{A} / \mathrm{C}$ simulation platforms, flexible and sustainable methods for model and simulator development, reuse, maintenance, documentation, and $\mathrm{V} \& \mathrm{~V}$ are required. The Modelica language [2] was chosen by Saab for A/C vehicle system modeling based on these premises [3]. Saab has been conducting research continuously to improve upon the used methods for model and simulator credibility assessment. Three recent projects are Modeling and Simulation for the 2010s Energy Management Systems [4], Validation of Complex Simulation Models [5], and Model Validation - from Concept to Product [6]. A key result of the project Validation of Complex Simulation Models was the formulation of the Saab Aeronautics Handbook for Development of Simulation Models [7]. This handbook covers the steps that a model developer needs to take to ready a model for simulator use. The focus of the first two subsequent projects were primarily on model development and $\mathrm{V} \& \mathrm{~V}$ during early development phases. Moreover, to achieve a complete methodology, all phases of model and simulator development need to be covered in detail.

The focus of the work presented here is on aspects that arise once nominal versions of the A/C models, simulators, and Hardware $(\mathrm{H} / \mathrm{W})$ testing stations have been produced. In these later phases of detailed design [8], the analysis shifts towards Software $(\mathrm{S} / \mathrm{W})$ and $\mathrm{H} / \mathrm{W}$ verification, enhancing sub-system and overall performance, fault analysis and diagnosis, as well as training and flight-test decision support. This type of use spans the entire product life cycle and the comprising techniques and methodology need to be able to survive during the rapid evolution of computational $\mathrm{H} / \mathrm{W}$ and $\mathrm{S} / \mathrm{W}$. The means of mitigation is to rely upon standards for information exchange, and automated model validation at all M\&S levels.

In the context of industry 4.0, the notion of Digital Twins is emerging as a popular concept $[9,10,11]$. Bondani and Bacchiega describes digital twins as

real-time digital replicas of a physical devices [12].

Dahman and Rossman provide a more general description where a digital twin 
is stated to

describes a virtual image of a real subject (human, application) or object (machine, environment) that reflects all relevant static and dynamic properties[11].

A digital twin is here interpreted as a modeled virtual substitute of a corresponding SoI which is executed alongside its physical counterpart. The virtual substitute is viewed as any simulation model or simulator mimicking selected aspects of the SoI. The digital twin utilizes data from the physical system in order to increase the knowledge of the SoI as well as the simulation application and its corresponding development methodology. A simulation application implemented in the $\mathrm{V} \& \mathrm{~V}$ framework outlined in Chapter 5 is considered a digital twin. As such, the work presented in this thesis contributes to the implementation and exploitation of aircraft sub-system digital twins.

Furthermore, this thesis covers the work conducted within the frame of the ITEA3 project Open Cyber-Physical System Model-Driven Certified Development (OpenCPS) [13] in evaluating and influencing the SotA in standardized co-simulation, simulator exchange, and automated model validation in later development phases. The chronologically earlier work of the thesis was conducted within the frame of the Nationellt Flygtekniskt Forskningsprogram (NFFP) project Model Validation - from Concept to Product [6].

\subsection{Aim and Research Questions}

The aim of this thesis is to expand current SotA towards increased industrial applicability in robust and efficient co-simulation, exchange of information between M\&S domains, and automated model validation and simulator credibility assessment. This is done by means of testing, evaluating, and influencing, applicable standards and techniques. The research questions that the presented work aims to answer are listed below.

RQ1 Is the Functional Mock-up Interface (FMI) standard applicable to the aeronautical industry and $\mathrm{A} / \mathrm{C}$ vehicle system development?

RQ2 Can multiple connected A/C sub-system be simulated in parallel without affecting accuracy and numerical stability?

RQ3 What aspects need to be addressed if model validation in later development phases is to be automated?

RQ4 How can the credibility information of $\mathrm{A} / \mathrm{C}$ sub-system simulation models be propagated to simulator level? 


\subsection{Delimitations}

The FMI standard is used to export models for execution outside of their original development environment. Other comparable standards exist, see Chapter 3. However, as a result of the modeling tools used at Saab, FMI is the obvious choice as the corresponding tool vendors support and push for the development of FMI. The thesis is delimited to the recently released System Structure and Parameterization (SSP) standard for the standardized exchange of simulators for equivalent reasons.

This thesis is delimited to Model Based System Engineering (MBSE) [14] methods employing executable simulation models mimicking A/C sub-systems; as well as establishing interoperability between architectural modeling and the aforementioned simulation models. Other types of simulation models exist in the fields of, for example, Computational Fluid Dynamics (CFD), computational mechanics, and software development. Even though the interoperability between the software development method and language Executable UML (xtUML) and systems simulation is demonstrated within the frame of the OpenCPS project [15], it is omitted from the scope of this thesis.

Achieving stable and parallel simulations of simulators is here achieved by means of the Transmission Line Method (TLM). Various other means to address the issue exist, see Chapter 4, and a selected few are mentioned for context. One such recent development is the energy preserving filter proposed by Benedikt et al. [16].

\subsection{Method}

The research presented here was conducted by implementing the Industry-aslaboratory methodology $[17,18]$. This methodology is an approach that is well suited to research collaboration projects between industry and academia. Industry-as-laboratory highlights the relevance of industrial input to academic research in order to ensure focus on industrially relevant engineering methods. The schematic description of Industry-as-laboratory presented by Muller et al. is clarified with respect to the context of the research presented here, see Figure 1.1. The presented methodology has previously been used in several similar research project constellations $[6,19]$. 


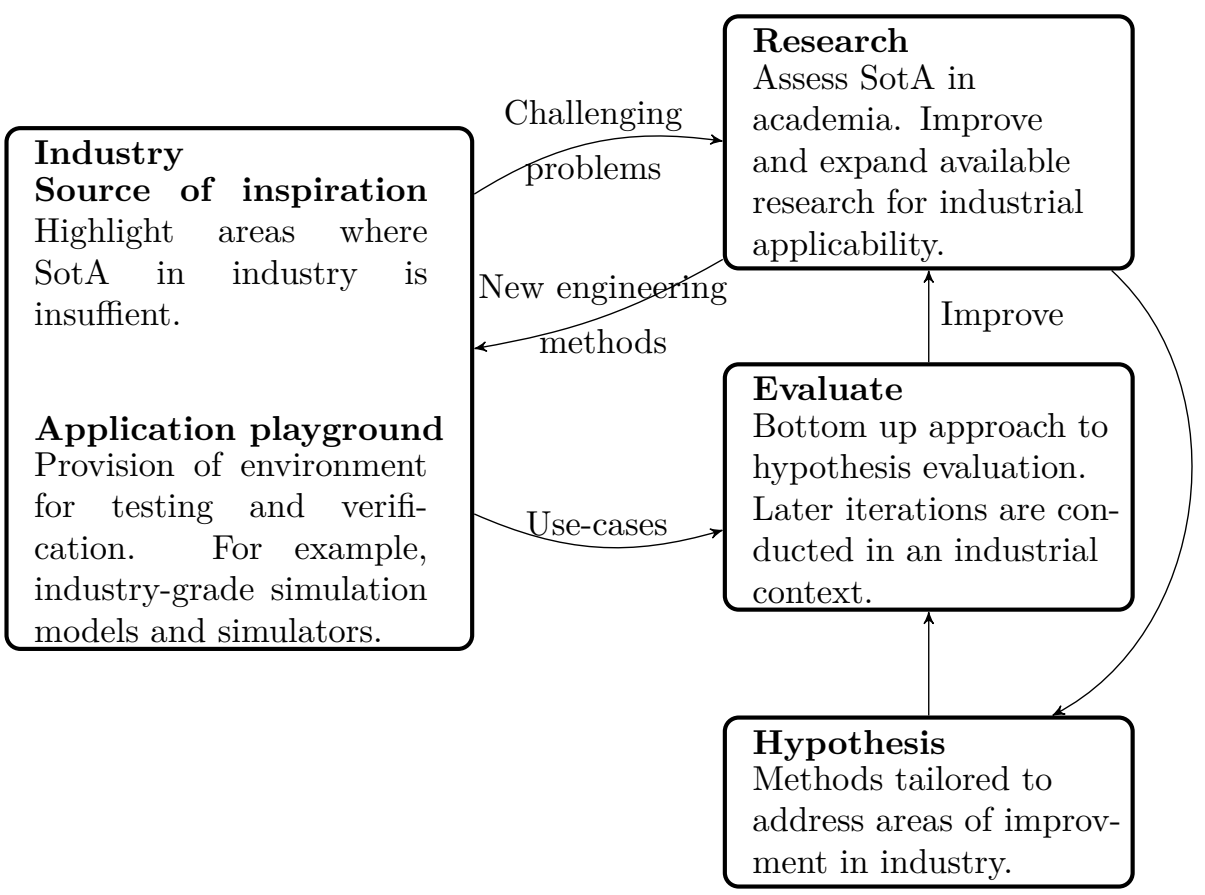

Figure 1.1 The industry-as-laboratory research approach. The method description is modified slightly compared to the illustration provided by Potts et al.[17] to highlight the connection to the presented research.

\subsection{Overview of Appended Papers}

Paper [III] propose methods for adapting physics-based simulation models of coupled A/C sub-systems to sampled co-simulation via the TLM technique and FMI standard. These techniques are applied on successively expanded industry grade application examples in [VI], [IV] and [VII]. In papers [IV] and [VII], the benefits of aforementioned techniques are demonstrated via an industrially relevant study connecting Environmental Control System (ECS) performance to pilot thermal comfort.

V\&V and credibility assessment are considered at all stages. Papers $[\mathrm{I}]$ and [II] contribute with methods that are applicable to all levels of simulation considered in the thesis. Paper [II] focuses on steady-state identification for automated historical data validation purposes on sub-system model level whereas Paper [I] targets propagating sub-system model assessed validity to simulator level, enabling on-line simulator credibility assessment. 


\subsection{Thesis Outline}

This thesis is presented from a top-down perspective. Chapter 2 presents the motivation, context, and coupling between the subsequent Chapter 3, Chapter 4, and Chapter 5. In Chapter 3, the utilized standards are introduced along with a simple example demonstrating the established engineering domain interoperability. Chapter 4 elaborates upon the work presented in Paper [III] and Paper [IV] and Chapter 5 on Paper [I] and Paper [II]. Both Chapter 4 and Chapter 5 begin with an introduction and theoretical overview of the respective topics. The contributions of the corresponding papers are then detailed in the latter sections of each chapter. The thesis is summarized and concluded, with answers to the research questions, in Chapter 6 . The thesis outline is visualized in Figure 1.2.

\subsection{Contribution}

The work has contributed in deriving an open specification of industrial requirements for co-simulation frameworks [20] which is available to all tool vendors supplying FMI supporting master simulation algorithms. In addition, the work has contributed to an accepted FMI standard change proposal adding callbacks, to FMI, for numerical stability reasons. The standard update is planned to be included in the coming FMI 3.0 under the heading Intermediate Variable Access [21].

An industry grade A/C vehicle systems simulator is developed and possible uses and benefits of such a mid-scale simulator, see Chapter 2, are demonstrated. This particular simulator is used to develop and demonstrate interoperability between various engineering domains such as $\mathrm{S} / \mathrm{W}$ modeling, $\mathrm{H} / \mathrm{W}$ modeling, architecture modeling, and modeling of human factors. The simulator is used for continuous bench-marking, capturing implicit requirements on, and testing of the FMI based simulation framework developed within the frame of the OpenCPS project, the OMSimulator [VIII].

A framework for automated validation and credibility assessment of aircraft vehicle system simulation models and simulators is outlined. Challenges are identified and a selected few are addressed. This includes the evaluation of methods for steady state-identification and propagating model level V\&V information to simulator level. 
Chapter 1: Introduction

Chapter 2: Simulator Applications in Aircraft Vehicle Systems Development Context and motivation

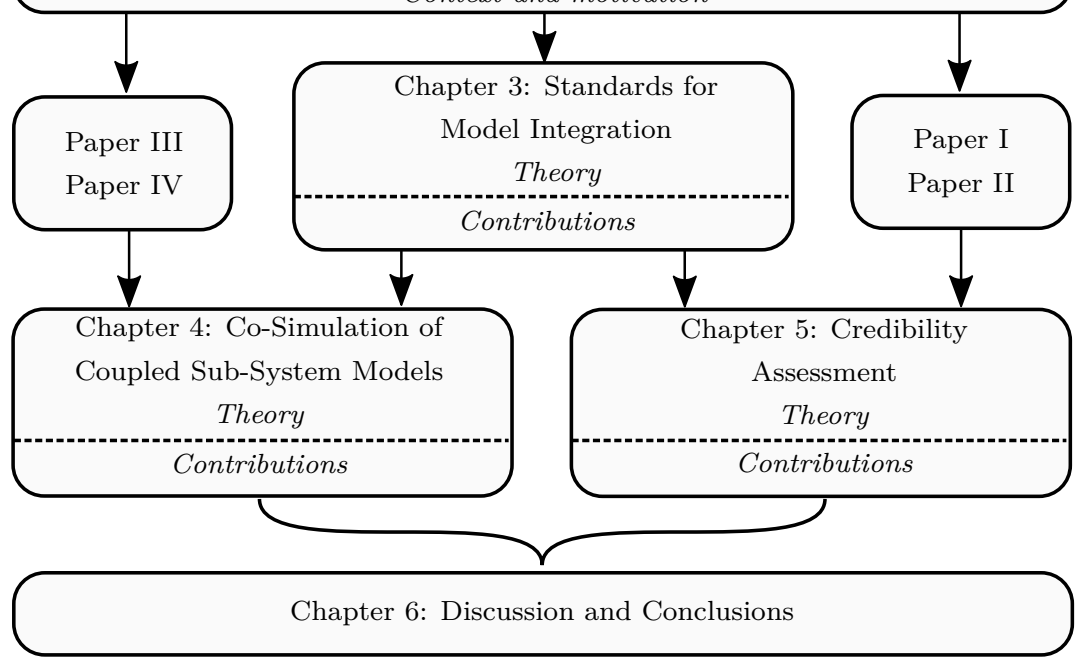

Figure 1.2 Thesis outline. Schematic representation of how the thesis chapters and appended papers relate to one and another. 


\section{2 \\ Simulator Applications in Aircraft Vehicle Systems Development}

The focus of this thesis is on methods for developing aircraft vehicle systems. In modern A/C, such as the Saab Gripen fighter, each included aircraft vehicle system consists of both $\mathrm{H} / \mathrm{W}$ and $\mathrm{S} / \mathrm{W}$ parts developed in completely different engineering domains. A particular emphasis is placed on the development and credibility assessment of simulators representing interconnected aircraft vehicle system along with selected parts of their surroundings.

Aircraft vehicle systems are sub-systems that exist in most A/C, both military and civil. Aircraft vehicle systems provide the principal A/C functionality that is essential for the most basic operation. Examples of such functionality are the provision of fuel to the engine via the incorporated fuel system, the control of aircraft control surfaces via the hydraulic systems, the pressurization of the cockpit via the environmental control system, etc. A complete list of the systems classified as aircraft vehicle systems in the Saab Gripen fighter, see Figure 2.1, is provided for context: pilot equipment, rescue equipment, fuel system, hydraulic system, electrical system, environmental control system, auxiliary power, and landing gears.

Furthermore, aircraft vehicle systems are merely a sub-set of the complete 


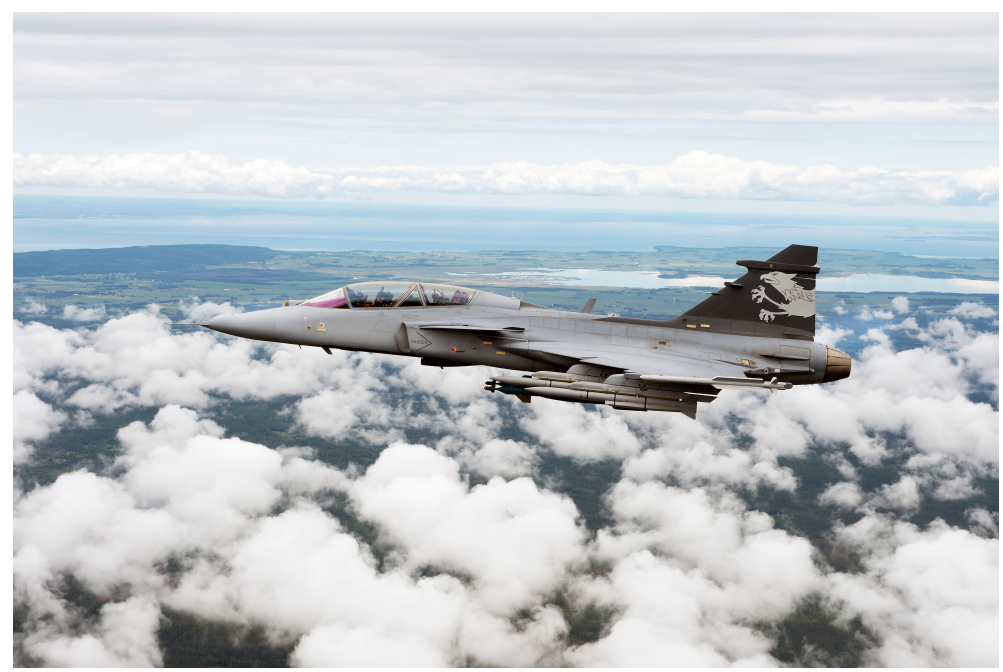

Figure 2.1 In flight photo of the Saab developed Gripen E demonstrator aircraft 39-7. Photo: courtesy of Stefan Kalm, Copyright Saab AB.

domain of applicability because the presented methods and techniques are general to a wide range of domains. Even so, this chapter provides a background to the methods currently put into practice at Saab Aeronautics for aircraft vehicle systems development. The aim is to illustrate the relevance of the research conducted by means of identifying strengths and weaknesses in the current way of working, as well as the stipulated improvements with the thesis results.

\subsection{Concepts and Terminology}

A simulator is here defined according to Definition 2.1.1 which is largely in line with the definition provided by Andersson et al. in [7].

Definition 2.1.1. A simulator is a platform for predicting the behavior, or verifying functionality, of coupled sub-systems using corresponding models originating in different engineering domains.

Definition 2.1.1 includes the notion of model. The definition of a model provided by Fritzon in [2] is adopted and presented in Definition 2.1.2. The applied experiment is explained as the process of exciting the model inputs to extract the sought information. 
Definition 2.1.2. A model of a system is anything an "experiment" can be applied to in order to answer questions about the system.

Both Fritzon and Ljung et al. [22] partition models into four different categories: mental, verbal, physical, and mathematical. The simulation models that are the primary focus here are of the mathematical sort. Here, simulation models are executable representations, able to describe the dynamic properties of the SoI via constituent Algebraic Equations (AEs) and Differential Equations (DEs) $[6,1]$. A different class of models considered in this thesis, which are not easily placed in any of the provided categories, are models describing System architecture. System architecture models are described by the National Aeronautics and Space Administration (NASA) [23] as

models that define the underlying structure and relationship of the elements of the system (e.g., H/W, S/W, humans in-the-loop, support personnel, communications, operations, etc.) and the basis for the partitioning of requirements into lower levels to the point that design work can be accomplished.

In other words, a system architecture model describes how the constituent sub-systems fit and function together to form a system at a higher level of abstraction. One relevant and obvious analogy is the relationship between simulation models and simulators.

\subsection{Modeling and Simulation of Aircraft Sub- Systems}

Various types of simulation models and simulators are used during the development of aircraft sub-systems. Eek [5, 6] and Steinkellner [4] provide overviews of various sub-system modeling techniques that are applicable during aircraft vehicle system development. Even though closely related, the focus here is instead on aircraft vehicle systems simulator development.

The question to be answered by the simulation governs which simulation application that should be used for the specific testing. If the purpose is to determine temperature and pressure spectra during transient conditions, then a detailed physics-based simulation model, connected to its corresponding controlling $\mathrm{S} / \mathrm{W}$, is likely to be required. However, if the purpose of the simulation is verify the activation of some specific $\mathrm{S} / \mathrm{W}$ logic during discrete changes in operating conditions, then simple simulation models consisting of various combinations of boolean statements may be sufficient. If the coupled dynamic behavior of several A/C sub-systems is under investigation, then coupled detailed models of the sub-systems in question are required. However, 
if the connections between the same sub-systems need to be verified, simple test stub models with accurate interfaces may be sufficient.

\subsubsection{Small-scale simulators}

Small-scale simulators are defined in Definition 2.2.1.

Definition 2.2.1. Small-scale simulators are simulation platforms that are intended for analysing one aircraft sub-system at a time, incorporating more than one M\&S domain.

At Saab, such simulation platforms are typically set-up using Hosted Simulation $[4,3]$. With hosted simulation, code is generated from one of the models developed to represent a particular aspect of the sub-system of interest. The second sub-system model, representing complementary aspects of the SoI, is kept in its original M\&S tool. The two sub-systems models are coupled, one in its original state and one as compiled external code, in the M\&S tool closest to the analysis to be conducted. One example of hosted simulation is the connection of an aircraft vehicle system $\mathrm{H} / \mathrm{W}$ model with its corresponding controlling S/W. Two small-scale simulators of such a sub-system are typically developed: one in the tool used for modeling of the included $\mathrm{H} / \mathrm{W}$ and one in the tool used for $\mathrm{S} / \mathrm{W}$ development. The former is used for fault and performance analysis, detailed $\mathrm{H} / \mathrm{W}$ design, mission analysis, etc. The latter is primarily used for control system development and $\mathrm{S} / \mathrm{W}$ verification. The main benefits of such platforms are that it is easy to modify the model that is in its original state, the $\mathrm{H} / \mathrm{W}$ model and $\mathrm{S} / \mathrm{W}$ developers are able to work in the domain specific tool that they are the most familiar with, and the exported models/code that is used in HIL test-rigs can be tested on a desktop at a small-scale. A small-scale simulator, intended for performance analysis etc. of the Gripen fighter ECS, is presented as an application example in [V].

\subsubsection{Mid-scale simulators}

Mid-scale simulators are simulators that include a few aircraft sub-systems. Mid-scale simulation is defined by Andersson in [24, 25] and similarly by Steinkellner in [4]. The definitions of Anderson and Steinkellner are slightly expanded, resulting in Definition 2.2.2.

Definition 2.2.2. Mid-scale simulation is the execution of a few coupled simulation models, developed in the best suited domain specific tools, that are 
jointly numerically complex enough to be executed more efficiently in a thirdparty tool.

The efficiency mentioned in Definition 2.2.2 needs to be established by means of weighing the benefits and drawbacks in terms of simulation accuracy and robustness, impacts on simulation execution time, Configuration Management $(\mathrm{CM})$, and the overhead costs associated with incorporating a third-party tool. Mid-scale simulators are here seen as a compromise, relevant when the required level of integration of a small-scale simulator is insufficient for the required detailed analysis that is too computationally expensive to be conducted using a large-scale simulator. Level of integration is here defined as a measure of how many of the A/C constituent sub-systems are units under test. As a result, methods for establishing mid-scale simulators need to require relatively little effort as the intended use, see Chapter 5 , of the simulation application tend to be quite narrow. Existing models need to be reused and integrated without affecting the validity assessed on a sub-system level. An example of a mid-scale simulator is presented in Figure 3.2 and Paper [IV].

\subsubsection{Large-scale simulators}

Large-scale simulators include most of the modeled aircraft's sub-systems along with their corresponding controlling $\mathrm{S} / \mathrm{W}$, either deployed on the actual $\mathrm{H} / \mathrm{W}$ or as models depending on the simulator and its pre-defined intended use(s). Large-scale simulators are defined in Definition 2.2.3.

Definition 2.2.3. Large-scale simulators are test-stations where several simulation models of the aircraft sub-systems are integrated and specific arrangements for performance or interoperability exist [24, 4].

As a result of the large-scale simulator size and the often high simulation performance requirements, the level of detail of the included sub-system models is often specified as low. This is an obvious trade-off that limits the possible reuse of available models and the feasible intended use(s) of the simulator.

Examples of large-scale simulators are the Mysim and Total system rig (T-rig) simulators. The MySim and T-rig testing stations are used extensively at Saab during the development of the Gripen fighter A/C. Mysim is a purely soft simulator without real-time requirements, used on desktops for early development testing during preliminary and detailed design. The T-rig is a real-time Hardware In the Loop (HIL) simulator that is used for development testing and formal verification of system requirements and flight safety. These two 
large-scale simulators are presented as application examples in Paper [I].

\subsubsection{Overview of simulator applications at Saab Aeronautics}

It is easy to perceive the covering of all required testing by a single parameterized simulator as beneficial from a maintenance and development perspective. According to Steinkellner,

Today's endeavor is a large master model that is gradually refined and used in any context [4].

However, even though reusing models and simulators is deemed as beneficial, the CM of the simulators and its included parts is increased with increased scope of the intended use $[1,26]$. The presented trade-off is not the only aspect limiting the reuse of simulation applications. In fact, Sivard states that

what to reuse and what to vary, becomes key knowledge

when reflecting on the topic of reusing assets in a product family [27]. One additional example is that high simulation speed is often of the essence even though real-time execution may not be required. Customer specific requirements as well as Intellectual Property (IP) and export control laws are other aspects that need to be considered. The benefits of model reuse considering the aspects listed above are described by Anderson et al. [26] and Lind et al. [3]. A work-flow for model development and export supporting the reuse of models is put into practice, to the extent possible with current SotA in industry, at Saab Aeronautics.

The simulation applications currently being used during vehicle systems development at Saab are schematically visualized in Figure 2.2a. Note that not all simulation applications are necessarily available during all phases of development. For example, early on in the development process of a new aircraft vehicle system, rigorous testing is conducted using small-scale simulators. Once the $\mathrm{H} / \mathrm{W}$ models and the corresponding $\mathrm{S} / \mathrm{W}$ are mature enough, the models are assembled together with interfacing modeled sub-systems into midor large-scale simulators.

The sizes of the circles in Figure 2.2 indicated the relative amount of testing conducted at each testing station. For example the fully virtual platforms, the small- and large-scale soft simulators, are depicted as large circles relative to the other representations because they are available to a broader set of engineers. The relative sizes between the circles may vary throughout development, the visualization represents an assessed nominal situation where all simulation 
applications are available. The vertical axis of the figure is labeled Level of representation, which here is a measure of how well the system represents the unit under test. A simulation model with accurate representation of both the true system dynamics as well as its statics has a higher level of representation than a steady-state simulation model of the SoI.

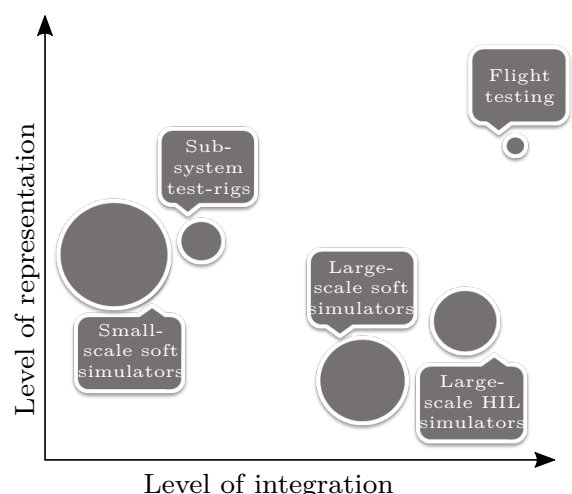

(a) Aircraft sub-systems test and development platforms currently available at Saab Aeronautics.

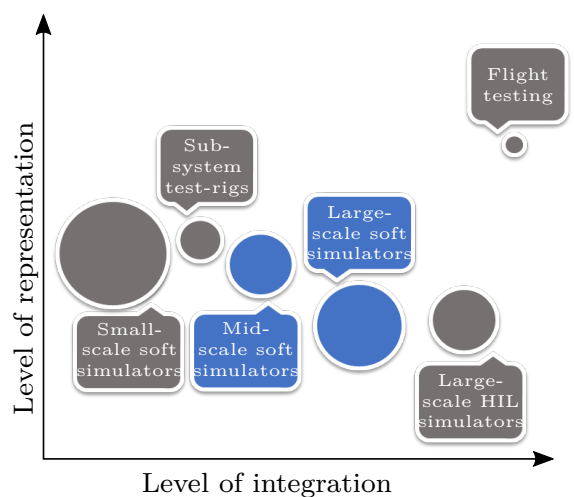

(b) Stipulated improvements on the available platforms are marked as blue.

Figure 2.2 Schematic visualization of the aircraft sub-systems development platforms available at Saab Aeronautics. The left hand figure represents the State of the art and the right hand figure the stipulated enhancements of the OpenCPS research results.

Refining the complete process of integrating models into simulators, presented in Section 2.3, allows for increased model reuse considering the domain specific tools used for aircraft vehicle systems development. This results in an increased level of representation for the large-scale soft simulators as well as the standardized development of mid-scale simulators. The stipulated improvements are visualized in Figure 2.2b. The large-scale soft simulators are stipulated to reach a higher level of representation as the available standards for model export allow for the use of more advanced solver methods during model execution in third-party $\mathrm{S} / \mathrm{W}$. This in turn enables large-scale simulation of sub-system models with an increased level of modeled detail. In addition, the development of mid-scale soft simulators is greatly simplified as existing small-scale simulators can be reused and assembled into study-specific mid-scale simulators. This enhancement motivates the development of such simulation applications and the feasibility is demonstrated in Paper [IV]. As the approach is to reuse existing small-scale simulators, and connecting them without introducing numerical errors, the level of representation of the constituent small-scale simulators is preserved in the mid-scale simulators. 


\subsection{Integrating Models into Simulators}

The complete process of deploying domain specific models in simulators is referred to as integration within the frame of this thesis. The verb integrate is interpreted according to the definition provided by the Merriam-Webster dictionary [28], see Definition 2.3.1.

Definition 2.3.1. Integrate: to form, coordinate, or blend into a functioning or unified whole.

More specifically, in a systems engineering context, the integration process is described by International Council on Systems Engineering (INCOSE) as the process to

synthesize a set of system elements into a realized system (product or service) that satisfies system requirements, architecture and design [29].

The latter description is in line with Definition 2.3.1. The process of integration is in [29] explained to be iterated alongside the $\mathrm{V} \& \mathrm{~V}$ process where the $\mathrm{V} \& \mathrm{~V}$ process is an activity that should be invoked as needed. V\&V is similarly incorporated into Definition 2.3.1. The unified whole is viewed as any simulator application, of which the validity is assessed according to a well established process, in which the constituent parts are the domain specific models. The $\mathrm{V} \& \mathrm{~V}$ process is ideally an independent process, conducted continuously, making the most of all currently available objective information. The functioning of a simulator is interpreted in light of the fact that the simulator needs to fulfil its intended use. To determine whether or not the simulator can be used as intended, its credibility as a whole needs to be established.

Figure 2.3 shows a general simulation application development process. Andersson and Carlsson et al. provide an overview of the constituent activities in [7] and [30]. The figure represents a simplification of the work-flow in current use at Saab Aeronautics. Here, the process is presented as general to three different simulation application levels: the Component level, the Model level, and the Simulator level. The component level refers to the modeling of components to be included in a larger sub-system model. A component could be, for example, a modeled cold air unit or cooling pack to be included in an ECS model [31]. The components are typically constructed using the founding building blocks of a preceding library level. This level is not depicted here; however, the process could very well be applicable for the development of such libraries. The model level refers to the development and use of a sub-system model; for example, 
any aircraft vehicle system model. As described previously, a simulator can be of varying size and complexity. The simulator level of Figure 2.3 refers to the development and use of any simulator in which two or more modeling domains are incorporated.

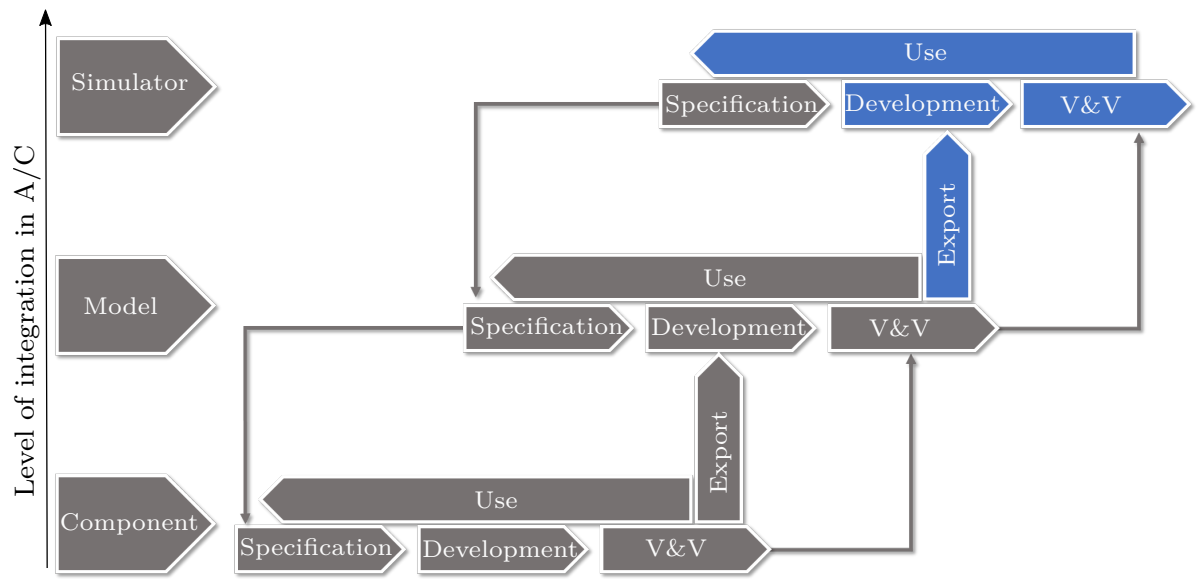

Figure 2.3 Simulation application development process. The application specification is described as a top down process whereas the $V \& V$ is described as a bottom up process. The fields highlighted in blue are targeted in the presented work as well as in the OpenCPS project.

The process of each level comprises five different activities: Specification, Development, $V \& V$, Export, and Use. The specification activity is formulated using input from the previous, and planned, use of all levels where the simulation application is included. In this step, the intended use of the simulation application is expressed; including a requirement specification, interface description, V\&V plan, etc. Both planned and current use need to be considered since the development is an iterative process. The actual modeling occurs during the development phase, which is preceded by an export activity of artifacts from a lower level of abstraction. At the component level, available library components are combined into a component model; at the model level, modeled components are connected into a sub-system model; at the simulator level, sub-system models from different domains are combined into a smallscale, mid-scale, or large-scale simulator. The final step of each level concerns $\mathrm{V} \& \mathrm{~V}$. In this step, suitable $\mathrm{V} \& \mathrm{~V}$ techniques are used to verify the specified requirements and to validate the simulation application's conformance to the SoI that it represents. A bottom up approach to $\mathrm{V} \& \mathrm{~V}$ is presented in the figure where the $\mathrm{V} \& \mathrm{~V}$ results are used as input to the corresponding process at the higher level of abstraction. Please note that $V \& V$ is a continuous process in the presented work-flow and not an activity that is only 
relevant once the application is complete. This is essential to achieve the sought after M\&S application cost efficiently [32]. The concepts of V\&V are discussed in detail in Chapter 5. One suggestion for how to realize the model and simulator validation activity shown in Figure 2.3 is presented in Figure 5.2.

The process of integrating models into simulators is described by the activities, specification, development, and V\&V of the simulator level in Figure 2.3. The export activity of the preceding model level results in import and assembly activities, here included in development, at the simulator level. 


\section{3 \\ Standards for Model Integration}

The administrative effort required for managing and integrating the models included in a simulator is large [4]. Aircraft sub-system development at Saab is therefore stipulated to benefit greatly from standardizing the model integration process. The use of open standards will allow for a wider selection of simulator execution S/W, here referred to as Master Simulation Tools (MSTs), potentially increasing the availability of simulators. Additionally, the introduction of standards is stipulated to help in reducing the manual effort associated with model integration, and therefore result in a more efficient process.

There are several different standards associated with the exchange and coupled simulation of models from different domains. The standards considered in this thesis are FMI [33, 34] and SSP [35, 36, 37]. The applicability, with respect to the aeronautical industry, of these standards is investigated and evaluated using both simple, see Figure 3.1 and Paper [III], and industry grade application examples [VI, IV]. The simple simulator example schematically visualized in Figure 3.1 is used as an aid in the following descriptions and discussions. The example is implemented as two simple Modelica models with signal type components from the Modelica Standard Library (MSL) [2]. MSL is a Modelica package containing fundamental modeling components, from several different engineering domains, developed and maintained by the Modelica Association (MA) [38]. 


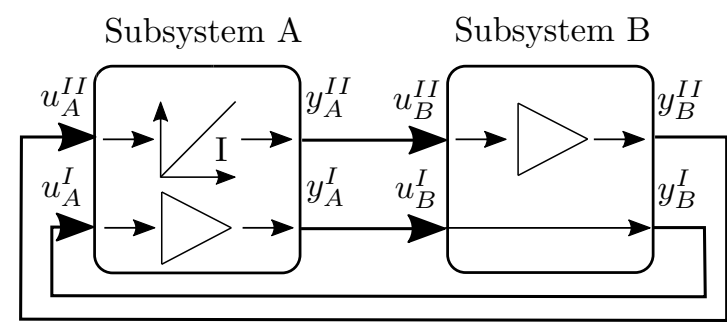

Figure 3.1 Two models, Subsystem A and Subsystem B, connected to form a small-scale simulator. This example is intended to be an aid when explaining the features of the FMI and SSP standards.

\subsection{Exchange of Models}

The FMI standard provides a standardized format for exporting and importing models for execution in an integrating environment that is different from the tool that is used for model development. Currently, over one hundred tools provide native support, to varying extents, for exchanging models according to the FMI specification. At the time of writing, FMI 2.0.1 [34] is the latest available version of the standard. However, the features and functionality of FMI 2.0 [33] is considered in this thesis as the latest version was released during the manuscript writing. FMI 2.0.1 is a maintenance release with no new features and the updates do not impact upon the presented work.

The modeling tools, supporting model export according to the FMI standard, in focus within the frame of this thesis are Dymola [39], OpenModelica [40] and Matlab/Simulink [41] for physics-based modeling, and Matlab/Simulink for the development of controlling S/W. Currently, Dymola and OpenModelica have native support for the export and import of Functional Mock-up Units (FMUs). Limited native FMI support is available from Simulink version R2017b; however, third-party S/W establishing support for both earlier and current tool versions are available, see for example [42] and [43].

A model exported according to the FMI standard is referred to as a Functional Mock-up Unit (FMU) in the specification. Models can be exported as FMUs for Co-Simulation (CS) or Model Exchange (ME). FMUs for CS are themselves responsible for integrating their comprising DEs via an included numerical solver. In ME FMUs this solver is omitted and it is the responsibility of the MST to numerically solve the model equations. A model exported as an FMU is packaged in a $<$ model $>$.fmu file. This file contains the model compiled binaries (or source code for source code FMUs) along with an Extensible Markup Language (XML) file denoted as ModelDescription. In short, the ModelDescription describes the model interface and properties according to a 
standardized XML schema.

One challenge that arises as a result of connecting co-simulation entities is the possible occurrence of algebraic loops and the ability to identify them. Algebraic loops, and the methods as how to address them, are described rigorously in the literature; see for example Kubler et al. [44] or Broman et al. [45]. An algebraic loop arises in a situation where a model input is directly dependent on one of its outputs. A common approach to dealing with algebraic loops is to iteratively find a consistent solution to the dependent inputs and outputs. Another approach is to incorporate non-physical delays. Such delays will break the loop; however, they will also alter the behavior of the simulator. A third approach, most relevant in simulator development, is to pass the responsibility for dealing with algebraic loops to the sub-system modelers. If the coupled sub-systems contain algebraic loops, then the wrong information is passed between the sub-system models. That being said, if available methods to deal with algebraic loops are to be used, their presence in a simulator need to be established.

All model internal dependencies are not necessarily exposed in black- or greybox models [46]. If these dependencies remain unexposed, then the presence of algebraic loops cannot be established. This issue is addressed in the FMI standard via the dependencies attribute which exposes the necessary internal dependencies to the ModelDescription file [33]. The ModelDesription file of a model of Subsystem A, see Figure 3.1, is provided in Appendix A. An algebraic loop will occur if Figure 3.1 is realized.

\subsection{Simulator Composition and Exchange}

The current SotA of model integration in the aeronautical industry is generally to use non-standardized and problem specific methods. Such methods are typically organization and tool specific and each simulator implementation is therefore bound to a specific MST. Solutions of that sort are typically difficult and expensive to maintain. Standardized methods to import and connect sub-system models are here seen as means to further favor efficient CM and the exchange of simulator applications. Such standardized methods will propagate the benefits of using a standardized modeling language [3] for sub-system modeling, such as Modelica, to simulator level.

High Level Architecture (HLA) is a standard for establishing interoperability between distributed simulation models, favoring model reuse and tool interoperability. The HLA standard has been under successive development since the early 1990's and can now be considered well established [47]. Three key concepts of the standard are: the Federation which describes the distributed 
simulation, the Federate which is the simulation entity, and the Run-Time Infrastructure (RTI) which manages the communication among the Federates $[48,49]$. Moreover, the HLA standard not only considers the assembly of simulators but also, much like the FMI standard, provides a standardized model format via specified functions that a simulation model needs to support to be considered a Federate. Even though interoperability between HLA and FMI was shown to be successful by Sievert among others [50], HLA is mentioned merely for context. The focus is instead placed on the recently released standard SSP [35] which aims to solve the same architecture specification problem. This delimitation is motivated by the standard stipulated tool support as it is a standard that is developed and maintained via the MA which is driven by the tool vendors and industrial parties also maintaining and improving on the FMI standard and Modelica modeling language.

The SSP standard is focused on connecting simulation models in a standardized manner, providing a nested hierarchical definition of included sub-systems. Where the FMI standard focuses on the exchange of domain specific models, the SSP standard provides a format for standardized connection, parameterization, and exchange of a connected set of models, i.e. a simulator application. The SSP standard specifies a XML schema used to carry this information. The simulator composition is stored in a format denoted System Structure Description (SSD). The corresponding < simulator $>$.ssd file is packaged along with its referenced resources in a $<$ simulator $>$.ssp file. Examples of SSD referenced resources are other SSP or SSD files and FMUs.

As the SSP standard is young, current tool support is scarce. However, support for SSP in the Unified Modeling Language (UML) [51] based modeling tool Papyrus [52] and OMSimulator was established during the OpenCPS project. These tools have been used to investigate and evaluate the standard in this thesis. The two M\&S tools, FMIComposer [53] and FMIGo [54], are mentioned for context as both tools provided FMI and SSP support early on. A brief overview of available open source and commercial FMI supporting MSTs are provided by Ochel et al. in [VIII].

\subsection{Interoperability with FMI and SSP}

The two sub-system models presented in Figure 3.1 are developed in Dymola and the corresponding simulation application is implemented as a Composite model in OMSimulator using the graphical editor OMEdit [55], see Figure 3.2a. A Composite model is a simulator in OMSimulator consisting of models exported from any FMI supporting modeling tool or a tool supported by tool-to-tool coupling [VIII]. An SSP file of the example constituent parts is exported from OMSimulator using the OMEdit Graphical User Interface (GUI). 
The example SSD file is presented in Appendix A. The SSP file is imported into Papyrus and a screen-shot of the resulting architecture is presented in Figure 3.2b. At Saab, S/W architecture and event-driven software is typically developed using tools incorporating variants of UML, such as Papyrus and BridgePoint [56]. The established interoperability connects simulator architectural modeling to the domain of systems simulation. Please note that SoI architecture modeling may occur at a different level of abstraction than simulator architecture modeling and these discrepancies need to be assessed and mapped to one another. For example, the system architecture model might state that there are dependencies between the models in Figure 3.1 but not provide information on signal level. This topic is elaborated upon in the OpenCPS project [57]; however, a final generic solution is not yet available.

Despite of the remaining challenges, the presented interoperability facilitates the further evolution of the MBSE methodology, where the models act as information carriers. Some of the benefits of MBSE, in identified by INCOSE in [29] are: 1) improved communication among development stakeholders, 2) increased ability to manage system complexity, 3) and improved product quality. The communication among development stakeholders is potentially improved significantly via the demonstrated interoperability. Architectural modelers are shown to have access to simulators in a development tool of their domain. Conversely, the architecture is communicated to the simulator developers and end users directly via the architecture model which is now part of the actual simulation application. 


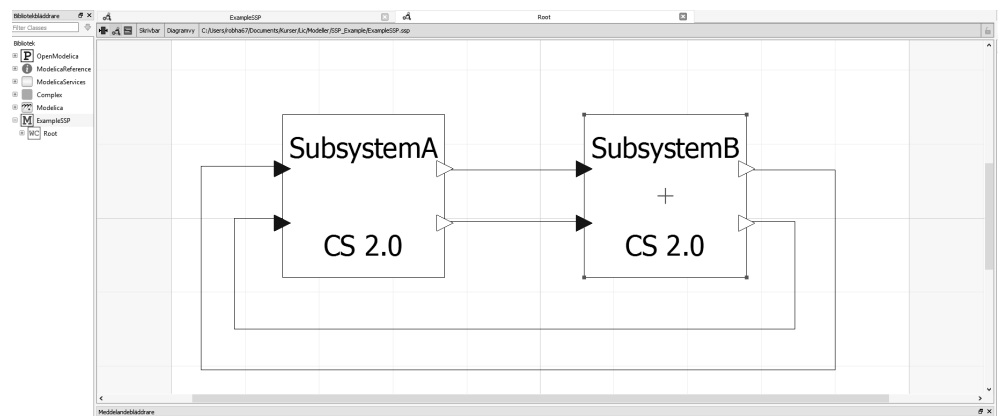

(a) The models of Figure 3.1 are imported into OMEdit. The model connections are established and the resulting Composite model is visualized in the figure. The composite model is exported from OMEdit as an SSP. The corresponding SSD file is provided in Appendix A.

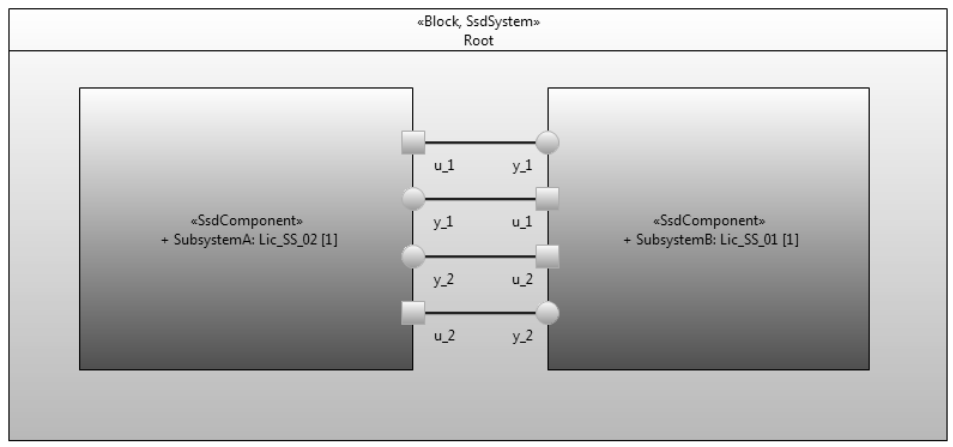

(b) Screenshot of SSP example imported into Papyrus.

Figure 3.2 Two simple Modelica models are exported from Dymola. The models are imported into OMEdit. The model connections are established according to specification, see Figure $3.2 \mathrm{a}$, and the configuration is imported into Papyrus, see Figure 3.2b. 


\section{4 \\ Co-Simulation of Coupled Sub-System Models}

Co-simulating any two coupled sub-system models means that the included models need to exchange information at discrete time instants, resulting in a sampled system. The definition of Co-Simulation (CS) provided in the FMI specification is provided here in Definition 4.0.1.

Definition 4.0.1. In $C S$, the data exchange between sub-systems is restricted to discrete communication points. In the time between two communication points, the sub-systems are solved independently from each other by their individual solver [33].

Sampled systems introduce challenges in terms of maintaining simulation stability and numerical accuracy. This chapter addresses these challenges within context of the simulation of coupled CS models. The FMI option of ME is not considered here. With FMI for ME, the solver is omitted from the exported model and the model equations are solved by the MST. The ME option is by no means irrelevant; however, the ability to use domain specific proprietary solvers enabled by the CS option is essential for many simulator applications. This is particularly true when reusing detailed legacy simulation models in simulator applications. Such models are often developed over the course of many years and they are typically, intentionally or unintentionally, tailored for optimal performance using the best suited solver of their corresponding development tool. 
A different view of the example provided in Figure 3.1 is provided in Figure 4.1. Figure 4.1 illustrates a small-scale simulator application that is subject to the challenges relevant for this chapter. The executions of a simulator with two incorporated and connected models, Subsystem A and Subsystem $\mathrm{B}$, is schematically visualized in the figure. A variable step-size solver, see for example Schierz et al. [58], is used to solve the Differential Algebraic Equations (DAEs) of Subsystem A, and a fixed step-size solver is implemented in Subsystem B. Information is exchanged between the models at equidistant time instants shown as vertical dashed lines. The sampling interval $T_{s}$ is the time between two adjacent communication points. The time at the simulator level is denoted as $T_{\text {macro }}$.

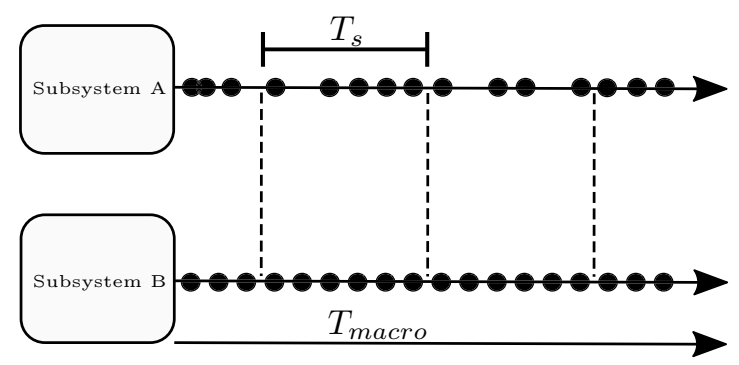

Figure 4.1 Two models connected to form a small-scale simulator. The models both have internal solvers solving the constituent DAEs. As a result, the MST merely passes data between the two incorporated models. A fixed-step solver is implemented in the Model Subsystem B and a variable-step solver in the Model Subsystem A.

\subsection{Challenges}

Different challenges arising as a result of sampling are identified, and elaborated upon, in the literature, see for example [16, 59, 60, 46]. A brief summary is provided here. Three key challenges associated with the simulation of coupled co-simulation models are: 1) the inevitable introduction of a phase shift, 2) the introduction of high-frequency content occurring as a result of discontinuities in the sampled signal, and 3) aliasing effects.

Figure 4.2 is presented as a basis for discussion concerning the first two listed implications of sampling. The continuous signal, dashed and red, in Figure $4.2 \mathrm{a}$ consists of two superimposed sinusoids with energy content at $1 \mathrm{~Hz}$ and $10 \mathrm{~Hz}$. This continuous signal is sampled with a frequency of $f_{s}=40 \mathrm{~Hz}$. Values between the sampling instants are kept constant using zero-order hold, type extrapolation, also referred to as constant extrapolation [III]. The sampled 
signal is presented as solid and black in Figure 4.2a. A Hamming window is applied to the time-domain data sequence prior to the Fast Fourier Transform (FFT) producing the frequency domain results presented in Figure $4.2 \mathrm{~b}$ and Figure 4.2c. The leakage occurring as a result of the windowing is reduced via the Hamming window [61].

The stability margins in connected sampled sub-system are generally decreased with an increase in $T_{s}$. According to Glad and Ljung [62], the sampling process can be approximated as a delay with transfer function

$$
G(s)=e^{-s \tau}
$$

for $T_{s}$ small in comparison to the signal energy content. In Equation 4.1, $0<\tau<T_{s}$. Such a delay corresponds to a phase shift of

$$
\phi(\omega)=\arg (G(s=i \omega))=-\omega \tau
$$

where $\omega$ is the angular frequency in radians per second. This phenomenon is visualized in Figure $4.2 \mathrm{a}$. The superimposed $10 \mathrm{~Hz}$ signal is shifted $0^{\circ}$ to $90^{\circ}$ throughout the sampling interval compared to the original signal, presented as dashed and red, which aligns with Equation 4.2 if $f_{s}=40 \mathrm{~Hz}$. The $1 \mathrm{~Hz}$ signal is shifted $0^{\circ}$ to $9^{\circ}$ degrees showing that the introduced phase shift increases with increased bandwidth of the sampled system. The exemplified phase shifts will impact upon the simulation results, and possibly result in an unstable simulation if not properly addressed.

The second topic for discussion, highlighted by Benedikt et al. [59], is the introduction of high-frequency content occurring as a result of discontinuities in the sampled signal. This phenomenon becomes apparent when comparing the frequency content of the two signals presented in Figure $4.2 \mathrm{~b}$ and Figure 4.2c. Note that the continuous sinusoid is sampled well above the Nyquist frequency, so the frequency domain distortion is not a result of aliasing. The frequency domain result shows that the sampling has introduced energy content at frequencies different than 1 and $10 \mathrm{~Hz}$. This energy content may excite high-frequency dynamics in a coupled sub-system that should not have been excited. This issue could be partly addressed by means of introducing low-pass filters on the inputs of a receiving model. However, the filter will remove energy content and introduce a phase shift, thereby altering the simulator.

If the sampling frequency is lower than twice the bandwidth of the sampled system, the output content will be distorted as a result of aliasing [62, 60]. Signal energy content above the Nyquist frequency will be mirrored around half of the sampling frequency and appear at an erroneous frequency. As a 


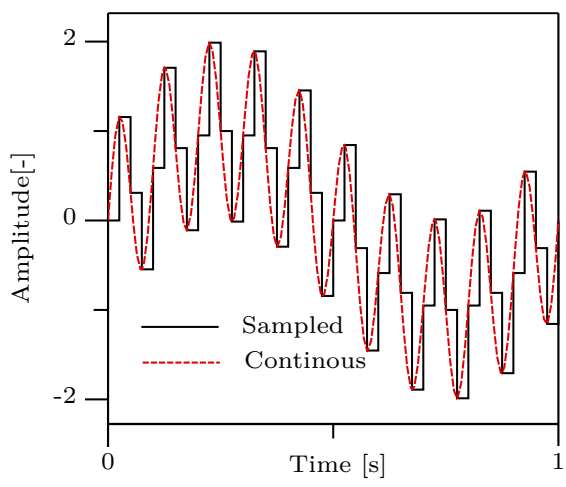

(a) Continuous and overlaid one and $10 \mathrm{~Hz}$ sinusoids in dashed red. Zero-order-hold sampling of the continuous signal in solid black. The sampling frequency is set as $40 \mathrm{~Hz}$.

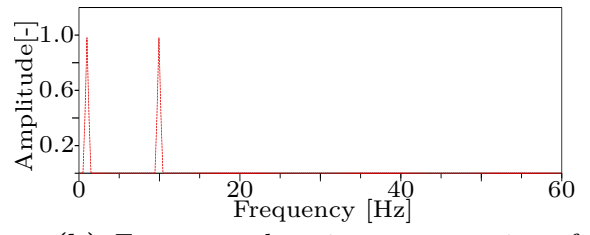

(b) Frequency domain representation of the continuous sinusoids of Figure 4.2a. Energy content is only present at 1 and $10 \mathrm{~Hz}$.

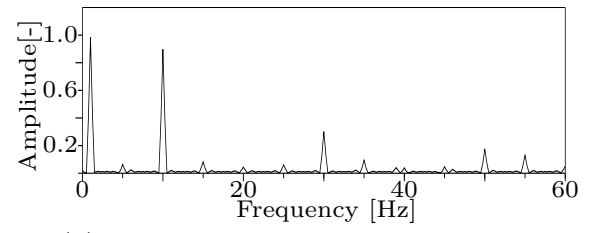

(c) Frequency domain representation of the sampled sinusoids of Figure 4.2a. Energy content at most shown frequencies.

Figure 4.2 Exemplification of two of the primary challenges of de-coupling coupled systems: 1) the inevitable introduction of a phase shift when sampling, and 2) the introduction of high frequency content occurring as a result of discontinuities in the sampled signal.

result, the simulation may become miss-representative or unstable. This could be partially addressed by means of adjusting the sub-system bandwidth via incorporated filters. Such filters will help to avoid aliasing, by means of removing signal content in risk of aliasing prior to sampling. However, in the end information is lost and this loss will impact upon the simulation results. It will then be up to the engineer or researcher to determine whether the result are usable or not, provided that the filtering itself did not result in an unstable simulation.

\subsection{Transmission Line Method (TLM)}

In Section 4.1, it is shown how the sampling of signals introduces delays. Such delays will at best impact upon the accuracy of the simulation results if not 
accounted for. However, information is propagated at a limited speed, a speed that depends on the considered physical domain. Such a physically motivated delay can be exploited to decouple models without compromising accuracy or stability [63]. TLM does precisely that. TLM, also known as Transmission Line Modeling or bi-lateral delay lines, was introduced during the 1960's by Auslander et al. [64] and later refined by Johns et al. in 1980 [65]. One of the significant contributions by Johns et al. was the introduction of numerical parasitic components as a means of assessing errors occurring as a result of discrepancies between utilized and physically motivated delays. In 1990, Krus et al. contributed with further improvements by means of introducing filtering in TLM type connections in order to address resonances in ideally modeled transmission lines [66]. The TLM technique has since then been implemented in various M\&S tools such as Hopsan [66, 67, 68], OpenModelica [69], and $B E A S T$ [70].

A schematic representation of a loss-less, hydraulic, and one-dimensional transmission line is provided in Figure 1. This transmission line is loss-less in the sense that there are no losses as a result of friction or contractions/expansions. Each end of the transmission line is subjected to a pressure $p_{i}$ and volumetric flow $q_{i}$. A wave will travel through the transmission line at the wave propagation speed, the speed of sound $a$. This means that a wave has passed through the pipe in $\Delta t_{T L M}=l_{\text {pipe }} / a$ seconds.

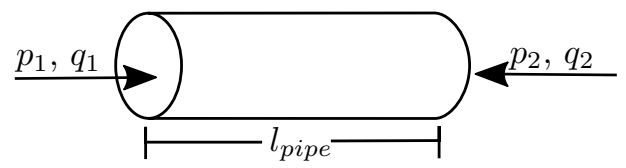

Figure 4.3 One dimensional, loss-less, hydraulic transmission line modeled as a pipe with length $l_{\text {pipe }}$. Each side of the transmission line is input a pressure and a volumetric flow.

The TLM equations describing such a transmission line are

$$
\begin{aligned}
& p_{1}(t)=Z_{c} \cdot q_{1}(t)+c_{1}(t) \\
& p_{2}(t)=Z_{c} \cdot q_{2}(t)+c_{2}(t)
\end{aligned}
$$

where

$$
\begin{aligned}
& c_{1}(t)=p_{2}\left(t-\Delta t_{T L M}\right)+Z_{c} \cdot q_{2}\left(t-\Delta t_{T L M}\right) \\
& c_{2}(t)=p_{1}\left(t-\Delta t_{T L M}\right)+Z_{c} \cdot q_{1}\left(t-\Delta t_{T L M}\right) .
\end{aligned}
$$

The variables $c_{i}$ in Equation 4.4 are known as wave variables. The wave variables collect the information at the opposite side of the transmission line at 
time $t-\Delta t_{T L M}$. The acoustic characteristic impedance $Z_{c}$ is the complex valued frequency domain ratio between input pressure and flow of an infinitely long transmission line, see for example Boden et al. [71]. It is related to the physical properties of the transmission line according to

$$
Z_{c}=\frac{\beta \Delta t_{T L M}}{V}
$$

if $\Delta t_{T L M}=l_{\text {pipe }} / a$ is inserted into the definition of characteristic acoustic impedance, see Equation B.2. In Equation 4.5, $\beta$ is the bulk modulus of the fluid,

$$
\beta=-V \frac{\partial p}{\partial V},
$$

a measure of how resistant the medium is to compression. The quantity $V$ in Equation 4.5 and Equation 4.6 is the volume of the transmission line.

The TLM equations for a loss-less hydraulic transmission line are derived, starting with Newton's second law and the continuity equation, in Appendix A for clarity and completeness. The TLM equations for other physical domains can be derived in an analogous manner; see, for example, Fritzon et al. for a derivation of the mechanical domain TLM equations [70].

\subsection{Transmission Line Characteristics}

A loss-less transmission line without inductance and delay is here referred to as an ideal capacitance. The pressure in such a capacitance is traditionally modeled as proportional to the integral of the net volumetric flow:

$$
p_{e}=K \int_{t_{0}}^{t} q_{n e t} d t
$$

where $q_{n e t}=q_{1}+q_{2}$ and $p_{e}(t)=p(t)-p\left(t_{0}\right)[69, \mathrm{VI}]$. The relationship between the constant $K$ and the TLM parameters $Z_{c}$ and $\Delta t_{T L M}$ can be derived starting with the definition of the bulk modulus. Equation 4.6 can be re-written as

$$
\frac{\partial p}{\partial t}=\frac{\beta}{V} q_{n e t}
$$

if the volume flow is defined as positive inwards, i.e $q_{n e t}=-\frac{\partial V}{\partial t}$, and both sides are divided by $\partial t$. Integrating Equation 4.8 with respect to time yields

$$
p_{e}(t)=\frac{\beta}{V} \int_{t_{0}}^{t} q_{n e t} d t .
$$


If Equation 4.5 is inserted into Equation 4.9,

$$
p_{e}(t)=\frac{Z_{c}}{\Delta t_{T L M}} \int_{t_{0}}^{t} q_{n e t} d t,
$$

then the relation to characteristic impedance and transmission line delay time becomes evident. In models developed using the Modelica language, delays (if relevant for the analysis) can be added via the Modelica delay operator between cascaded modeled capacitances.

Taking the Laplace transform of Equation 4.10 yields

$$
P(s)=\frac{Z_{c}}{s \Delta t_{T L M}} Q_{n e t}(s)
$$

if $q_{n e t}\left(t_{0}\right)=0$.

The TLM equations, see Equation 4.3 and Equation 4.4, provide a relationship between pressure and flow at the two ends of a transmission line. If the transmission line is assumed to have one closed end, then the net volumetric flow can be related to pressure and a transfer function on the same format as Equation 4.11 can be derived. So, inserting $q_{2}(t)=0$ into Equation 4.3 and Equation 4.4 yields

$$
p_{1}(t)=Z_{c} q_{1}(t)+p_{2}\left(t-\Delta t_{T L M}\right)
$$

and

$$
p_{2}(t)=Z_{c} q_{1}\left(t-\Delta t_{t l m}\right)+p_{1}\left(t-\Delta t_{T L M}\right) .
$$

Inserting Equation 4.13 into Equation 4.12, taking the Laplace transform, and solving for $P_{1}(s)$ results in

$$
P_{1}(s)=Z_{c} \frac{1+e^{-2 \Delta t_{T L M}}}{1-e^{-2 \Delta t_{T L M}}} Q_{n e t}(s)
$$

where $Q_{n e t}(s)=Q_{1}(s)$ as $Q_{2}(s)=0$.

In Figure 4.4, the frequency response transfer function magnitudes of Equation 4.11 and Equation 4.14 are plotted for a pipe with diameter $d=0.1 \mathrm{~m}$ in which air is flowing. The length of the pipe is set as $l_{\text {pipe }}=5 \mathrm{~m}$ in Figure $4.4 \mathrm{a}$ and it is varied in Figure 4.4b. Figure 4.4a shows how an ideal hydraulic capacitance differs from a transmission line. The two representations have identical static gains between net flow and pressure. However, the pipe resonance frequencies are captured by the transmission line representation whereas they are not by an ideal capacitance. Figure $4.4 \mathrm{~b}$ illustrates how the resonance frequencies change with changing transmission line length; an increase in transmission-line 


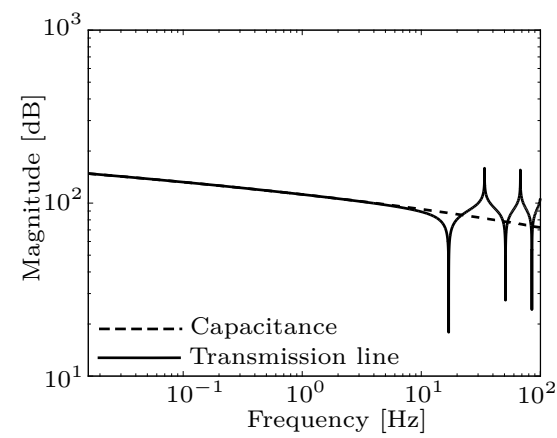

(a) Frequency domain magnitude comparison of an ideal hydraulic capacitance, see Equation 4.11, and transmission line, see Equation 4.14, for $l_{\text {pipe }}=5 \mathrm{~m}$.

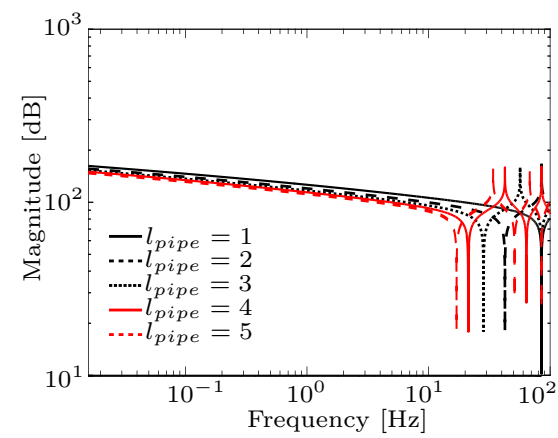

(b) Frequency response magnitude of closed end transmission line transfer function, see Equation 4.14, for transmission line lengths of $1,2,3,4$, and $5 \mathrm{~m}$.

Figure 4.4 Frequency domain characteristics of loss-less hydraulic transmission line and an ideal hydraulic capacitance. The characteristic impedance is specified as $Z_{c}=37823 \mathrm{sPa} / \mathrm{m}^{3}$ corresponding to a pipe with diameter $d=0.1 \mathrm{~m}$ in which air is flowing.

length results in a lower resonance frequency.

TLM exploits the characteristics of transmission lines to derive an independence between coupled models. In principle, a modeled transmission line is extracted from one or both models to be connected via a physical connection. This transmission line is positioned in the actual connection. In that way, the properties of the sampling procedure are encompassed by the transmission line and the sampling procedure does not introduce an error provided that the sampling interval is correctly related to $\Delta t_{T L M}$ and that the characteristic impedance can be assumed to be constant. This is not true for an ideal hydraulic capacitance because such a capacitance does not include a delay between the two sides of the pipe. A phase shift is introduced between flow and pressure, but it is not equivalent to that of a delay.

\subsection{TLM and FMI}

Neither FMI 2.0 or FMI 2.0.1 are adapted to support physical connections in general or TLM specifically. In Paper [III], various solution alternatives combining FMI and TLM are investigated and evaluated. As a result, a strategy for asynchronous simulation of FMUs, coupled via TLM-type physical connections, is formulated. Asynchronous here means that each TLM connection has its own independent time delay. Consequently, each included FMU has its own maximum allowed sampling interval. This can be compared to the syn- 
chronous algorithm presented in [64]. Auslander et al. propose a method for simulating models of coupled transmission lines where the transmission lines are grouped to form transmission lines with equal delay times. These lines are then decoupled for an equal amount of time and a synchronous exchange of information between groups does not result in modeling errors. The FMI supporting tool Hopsan incorporates a similar approach with synchronized exchange of data; however, the transmission lines are not grouped. Instead, a transparent modeling error is accepted to enable full parallelization. This is an efficient approach for simulating models in the TLM supporting tool where they were originally developed. However, in an FMI supporting MST, the option of asynchronous communication without the introduction of modeling errors can be essential depending on the M\&S activity. The frequently used detailed simulation models are often not robust enough to handle significant coupling errors.

Multi-domain co-simulation is bound by the parallelization techniques, on a model level, supplied by the model development tools. An MST can therefore only exploit decoupling strategies for parallelization at the system level. Clustering FMUs with equal coupling delays to avoid modeling errors is feasible. However, the parallelization benefits of small-scale and mid-scale simulators will be small with such an approach as they typically only consist of a small number of FMUs. Asynchronous communication is here seen as a more efficient approach because each incorporated FMU can be executed in parallel without the introduction of numerical errors. Asynchronous communication between FMUs connected via transmission lines is implemented in the OMSimulator [VIII].

\subsubsection{Accessing interpolation data}

The SotA in standardized co-simulation is to let the input values remain constant throughout the sampling interval; an approach known as constant extrapolation or zero-order-hold, see Figure 4.5a. The challenges associated with sampled sub-system models are the most prolific in such situations and great knowledge of the incorporated models is required to ensure stability and accuracy. With TLM, interpolation of input data is possible. Therefore, suitable interpolation strategies need to be selected. The current format of the FMI standard does not allow FMUs to access data at arbitrary time instants, but only at the beginning of each sampling interval $T_{s}$. Three possible workarounds exist: 1) sub-stepping, 2) append interpolated time derivatives to the FMU inputs, and 3) inserting the entire input interpolation table into each FMU. The two latter workarounds are respectively categorized as coarsegrained interpolation and fine-grained interpolation in Paper [III]. The method of coarse grained interpolation is described in detail in Paper [III]. Focus here 
is placed on sub-stepping and fine-grained interpolation.

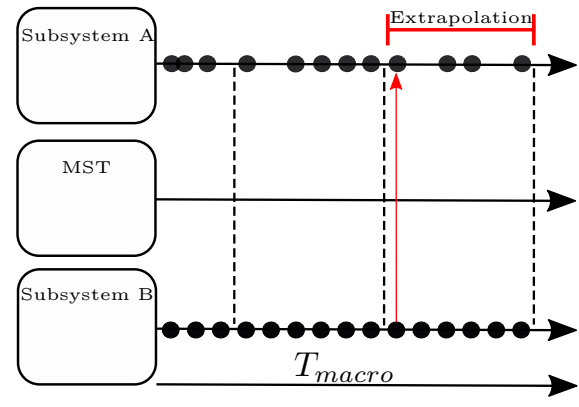

(a) SotA in standardized CS. Inputs are updated at the beginning of each sampling interval. Inputs for intermediate time instances are extrapolated, most commonly using constant extrapolation also known as zero-order-hold.

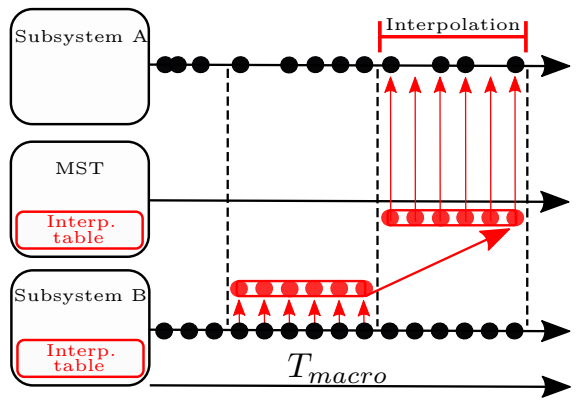

(b) Subsystem A is specified to be executed for a pre-defined number of substeps during each sampling interval. Intermediate input values can then be accessed from an interpolation table in the MST as updating FMU inputs is allowed at the beginning of each sub-step.

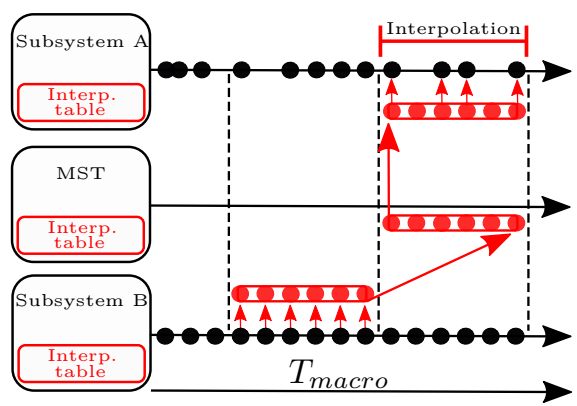

(c) An interpolation table is constructed in Subsystem B. This interpolation table is provided to Subsystem A via the MST. The Subsystem A model is wrapped with the necessary interpolation functionality.

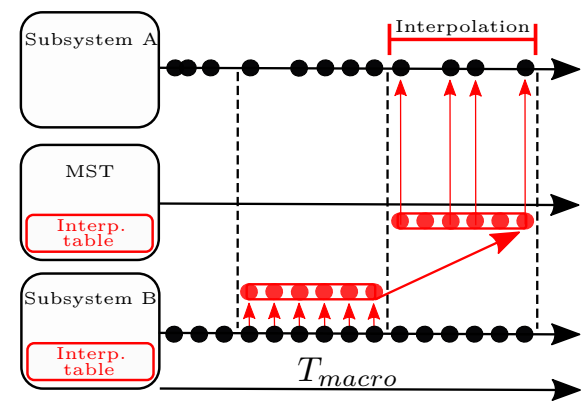

(d) The FMI standard is extended with functionality for setting FMU intermediate inputs. Subsystem A is allowed access to an interpolation table in the MST whenever the internal solver needs updated information.

Figure 4.5 Communication between connected FMUs. The flow of information from Subsystem A to Subsystem B is depicted as red. The converse flow of information is omitted from the figures for clarity. Figure 4.5a visualises the SotA in standardized CS. Figure 4.5b, Figure 4.5c, and Figure 4.5d aim to describe various solutions to establishing interoperability between FMI 2.0 and TLM. Subsystem B is depicted to output an interpolation table in the three latter figures. Sub-stepping Subsystem B or introducing callbacks for intermediate outputs and inputs are additional considered approaches; however, they are not represented in the figure. 
The methods of sub-stepping and fine-grained interpolation are visualized in Figure 4.5. In order to describe the principles, focus is directed towards the communication from Subsystem B to Subsystem A. However, note that bi-directional information is required if connecting models with TLM type connections, see Equation 4.3 and Equation 4.4. For a hydraulic transmission line, the volumetric flows of both Subsystem $A$ and Subsystem B are sent to the MST. The wave variables $c_{i}$ are computed in the MST via Equation 4.4. The pressures can then be calculated through Equation 4.3. All TLM methods presented in Figure 4.5 are depicted with Subsystem B supplying a fixed resolution interpolation table to the MST. This can be done, considering FMI 2.0, via a number of pre-defined sub-steps or by means of modifying the interface to include an output vector, of pre-defined resolution, along with a corresponding time vector.

The simplest and most straight forward workaround to establish interoperability between FMI and TLM is to force each FMU to do a number of sub-steps. The sub-steps allow the FMUs to access interpolated input values with the resolutions of the conducted sub-steps, see $4.5 \mathrm{~b}$. For models with fixed-step solvers, the solver step size can be synchronized with the sub-steps to ensure the passing of accurate input data. However, many industry grade physicsbased simulation models require variable step-size solvers with error control [58]. For CS FMUs with variable-step solvers, extrapolation is required for internal steps that are intermediate the sub-steps. So, even though an improvement compared to the SotA, such communication does not result in a fully accurate representation of the TLM connection and stability cannot be guaranteed. This particular method is tested and evaluated in [VI] and [72]. The results show that sub-stepping is not sufficient to maintain stability in all of the investigated examples. Additionally, implementing sub-steps may have a significant negative impact on the simulation execution time if invoked on models with variable step-size type solver as each sub-step results in discontinuities in the model inputs.

The second workaround is schematically visualized in Figure 4.5c. In Paper [III], fine-grained interpolation in FMUs is enabled by means of sending complete interpolation tables at the end of each sampling interval. In that case, an instance of the interpolation table is located inside both FMUs: Subsystem $A$ and Subsystem B. Consider data sent from Subsystem A to Subsystem B: Subsystem $A$ saves output values in an interpolation table of fixed resolution. Once a sampling interval is completed, the interpolation table is passed from Subsystem $A$ to Subsystem $B$ via the MST. Since the data is passed via a TLM type connection, the interpolation table is valid $\Delta t_{t l m}$ seconds into the future and Subsystem B can, as a result, interpolate the current values such that the internal solver has access to accurate inputs for all internal time steps. This particular approach is compatible with FMI 2.0 and it is evaluated in [III, IV]. 


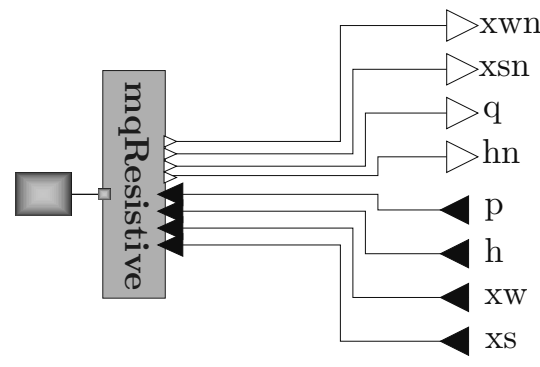

(a) De-grouping of Modelica model connector at interface prior to FMUexport.

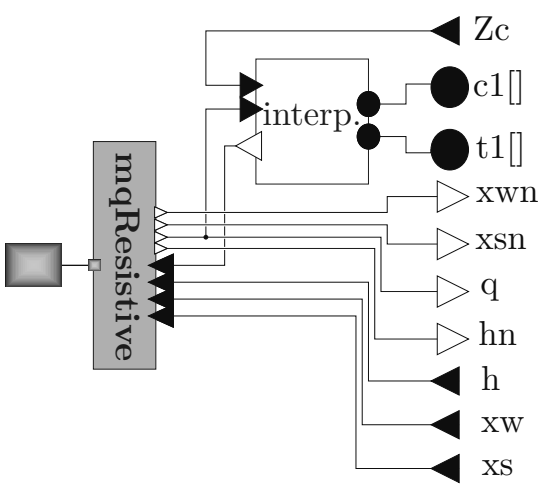

(b) Modelica model wrapper enabling TLM type connection with interpolation inside the FMU.

Figure 4.6 Modelica models of two different hydraulic types of interfaces. Figure 4.6a shows a simple conversion from a Modelica connector to passing data in the form of single inputs and outputs of fixed causality. The wrapper of Figure $4.6 \mathrm{~b}$ includes the interpolation functionality required to enable finegrained interpolation with the current version of FMI.

Drawbacks of fine-grained interpolation in the FMUs are the necessary modeling side modifications as well as the passing of entire interpolation tables at each communication point. In addition to extracting suitable transmission lines from each model to the MST, the constituent models need to be wrapped with interpolation functionality. An example of such a wrapper for a hydraulic interface of a Modelica model is provided in Figure 4.6b. The original interface is provided in Figure 4.6a. Originally, the pressure $p$ and the volumetric flow $q$ are passed like all other information that is relevant, for this particular model. The wrapper visualized in Figure $4.6 \mathrm{~b}$ is designed to accommodate a transmission line type of connection positioned in the MST with characteristic impedance $Z_{c}$, see Equation 4.5. This particular wrapper receives an interpolation table in the form of two vectors of pre-defined length: a vector of wave variables $c_{1}$, see Equation 4.4, and a vector of corresponding time stamps $t_{1}$. These two vectors are constructed in the MST given information from the interfacing model. The outputs $x_{w n}, x_{s n}$, and $h_{n}$ pass information on liquid phase water content, steam phase water content, and enthalpy to the interfacing model. The corresponding information is received from the opposite side via the inputs $x_{w}, x_{s}$, and $h$. These transport quantities are bypassed the TLM connection and they are simply passed on as delayed signals. The transport quantities time constant is related to the velocity of the flow which is assumed as significantly longer than $\Delta t_{t l m}$. 
Unlike in Figure 4.5c, the wrapper of Figure 4.6b does not construct and supply the MST with a high resolution vector of volume flow but simply the value at the end of each sampling interval $T_{s}$. Even so, the wrapper enables interpolation of input data via TLM compared to the extrapolation required for models with traditional physical interfaces.

To mitigate the drawbacks of fine-grained interpolation with FMI 2.0, new FMI functionality is proposed in Paper [III]. The accepted proposal is to introduce two new callback functions to the FMI standard. The callback functions allow CS FMUs to access and populate interpolation tables in the MST at time instances different than the communication points defined by the sampling intervals. In this way, high resolution input data is available to the CS FMU whenever it is needed by the internal solver. The proposed solution is schematically visualized in Figure 4.5d. A detailed description of the functionality is provided in Paper [III]. 
"The thing that doesn't fit is the thing that's the most interesting: the part that doesn't go according to what you expected."

Richard P. Feynman 


\section{Credibility Assessment}

\subsection{Concepts and Terminology}

Balci formulated one of the more popular definitions of $\mathrm{V} \& \mathrm{~V}$ as presented in Definition 5.1.1 [32].

Definition 5.1.1. 1) model validation is concerned with assessing whether the right model is built, and 2) model verification is concerned with assessing whether the model was built right.

More specifically, model validation is the task of determining whether the model represents the SoI well enough to fulfill its intended use(s); the importance of well defined intended uses is discussed by Balci and Ormsby in [73] and by Piersall and Grange in [74]. The validity assessment is conducted via validation metrics. Such metrics quantify the difference between the model and SoI System Response Quantities (SRQs) [75]. In contrast, model verification is the task of establishing whether the model has been transformed correctly, for example from specification to executable implementation. These definitions are generally agreed upon in the literature even though the wording might be different, see for example $[76,77,78]$.

$\mathrm{V} \& \mathrm{~V}$ are activities that influence the simulation result's credibility. Credibility assessment is a wider term incorporating aspects such as Uncertainty Quantification (UQ), use history, and people qualifications. NASA suggests a total of eight factors, along with guidelines on how to account for them, that contribute to M\&S credibility in the Credibility Assessment Scale (CAS), see Appendix B of [76]. Eek provides a thorough overview of $\mathrm{V} \& \mathrm{~V}$ and credibility assessment in [6]. 


\subsubsection{Domains of model operation}

A model Operational Domain (OD) is an $n$-dimensional volume spanned by all feasible values of model inputs affecting observable system states $[79, \mathrm{~V}]$. Such a domain can be defined once the model interfaces and its intended use(s) are specified. The model OD might be different from the OD of the SoI. If, for example, the SoI is a test rig able to run an aircraft vehicle system on the ground, then potential inputs such as Mach number and altitude remain constant in all conducted experiments. If the model of the same sub-system is specified to represent both the test-rig and the aircraft sub-system in an actual $\mathrm{A} / \mathrm{C}$, then the model OD will be of higher dimension.

In Figure 5.1, three different hypothetical ODs are visualized: $\mathrm{OD}_{\mathrm{SoI}}, \mathrm{OD}_{\mathrm{A}}$, and $\mathrm{OD}_{\mathrm{B}} \cdot \mathrm{OD}_{\mathrm{A}}$ and $\mathrm{OD}_{\mathrm{B}}$ are $\mathrm{ODs}$ of two different models of the same SoI; for example, two models with different level of detail as a result of different intended uses. The SoI that the models are designed to mimic has operational domain $\mathrm{OD}_{\mathrm{SoI}}$. Experiments on the SoI are conducted in the points jointly forming the corners of a convex hull demarcating the dark grey region denoted Domain of Validation (DoV). In two dimensions, a convex hull can be seen as the smallest area with convex faces that encloses a given set of points. If comparisons between the SoI and models are conducted in these operational points, then each model will have a DoV. DoV $\mathrm{B}_{\mathrm{B}}=\mathrm{DoV}$ as $\mathrm{OD}_{\mathrm{B}}$ encompasses the area denoted DoV. This is not the case for $\mathrm{OD}_{\mathrm{A}}$ and $\mathrm{DoV}_{\mathrm{A}}$ is a vertical line between the two leftmost experiment settings. Model $\mathrm{A}$ is not intended to represent the true system outside of its OD and comparisons most often cannot, and should typically not, be made at such settings.

An M\&S application is considered valid if it fulfills its intended use during specific operating conditions. However, as soon as the model is used under slightly different operating conditions, the validity can be questioned. Model $\mathrm{C}$ in Figure 5.1 can thus only be said to be valid in the four operational points where it has been compared to the SoI. However, most models are developed to predict system behavior at untested settings. The concept of validity thus need to be expanded to account for operation in the entire model OD. This can be done by means of incorporating a coverage metric. Such a coverage metric needs to distinguish between interpolation and extrapolation. In the context of conducting model based predictions, interpolation corresponds to conducting $\mathrm{M} \& S$ activities under model operating conditions located within the confines of the established DoV. In contrast to interpolation, extrapolation corresponds to conducting M\&S activities at untested model settings outside of the DoV. 


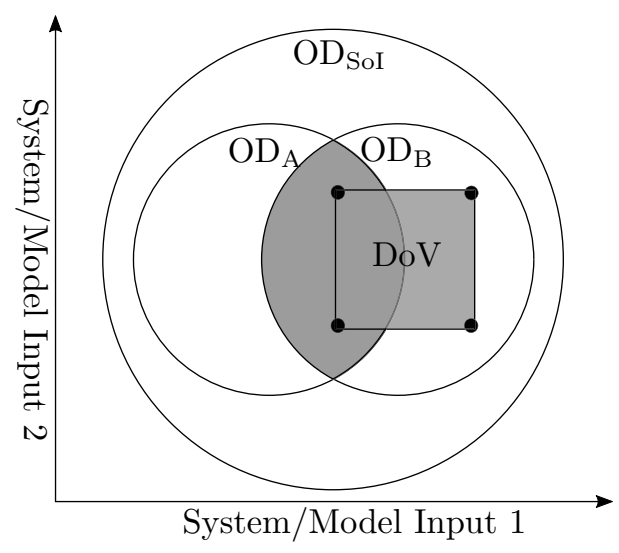

Figure 5.1 Visualization of the domains relevant to model and simulator credibility assessment. Three different Operational Domain (OD) are shown: the $O D$ of the System of Interest (SoI) is denoted $O D_{\text {SoI }}$, the OD of Model $A$ is denoted $O D_{A}$, and the $O D$ of Model $B$ is denoted $O D_{B}$. The domain demarcated by four conducted validation experiments is denoted DoV.

\subsubsection{Operational domain coverage}

Model coverage is a measure of how well a model's OD is covered by a given set of validation experiments. Oberkampf and Roy provides a comprehensive discussion on the notions of ODs, DoVs, comparisons between the two, and how they relate to making model based predictions [75]. Atamturktur et al. presents an objective coverage metric accounting for the addition of new validation experiments in [80], the nearest neighbor metric. In 2015, Atamturktur et al. formulated four criteria to which an exemplary coverage metric should be sensitive: 1) conducting validation experiments at an untested model operational point should always improve coverage; 2) diverse validation experiments yield better coverage than clustered validation settings; 3) regions of extrapolation result in a degradation of coverage; 4) the metric should be objective, [79]. As a result, the nearest neighbor metric was modified,

$$
\eta_{c}=1 / g \sum_{i=1}^{g} \min \left(d_{E, i}\right)+d_{Z I, i},
$$

to account for all of the specified criteria. The metric $\eta_{c}$ in Equation 5.1 decreases with increasing coverage of the OD. If every point within the OD is validated, then the metric is zero. The total number of grid points within the model's OD is denoted $g$. The coverage metric is normalized by $g$ ensuring that the metric remains unaffected by the resolution of the OD. The distance $d_{E, i}$ is the length from grid point $i$ to the closest validation point. The distance $d_{Z I, i}$ is the smallest distance from grid point $i$ to a convex hull spanned 
by the validation experiments. The distance $d_{Z I, i}$ is specified as zero for all grid points contained within the convex hull, thus serving as an extrapolation penalty as it penalizes clustered validation settings by means of incorporating the distance to the area of interpolation.

If such a metric is minimized for each selection of validation experiments, then there is no experiment better suited for model validation than the one selected. Equation 5.1 describes one of several existing coverage metrics, see for example [81]; however, the nearest neighbor coverage metric accounts for all the previously presented criteria.

A coverage metric, such as the nearest neighbor metric, only conveys information about coverage. This means that it does not in itself include information describing how well the model represents the SoI in the conducted validation experiments. However, experiments on how to append such information to the nearest coverage measure have been conducted, see Equation 5.2.

\subsection{Automating Model Validation}

In Paper [I], it is stated

The proposed method for assessing the credibility of a simulator is intended to make use of the results from $V \& V$ of the individual simulation models in a simulator, compile this information, and present it to simulator users in a clear and comprehensible manner.

A clearly expressed and highly automated approach to model V\&V is necessary if the method of simulator credibility assessment proposed in Paper [I] is to be feasible. Even though aspects such as independence in $\mathrm{V} \& \mathrm{~V}$ as well as the importance of conducting $\mathrm{V} \& \mathrm{~V}$ activities are stressed in the literature, see for example $[82,77]$, concrete proposals for how to automate V\&V of simulation models are rarely found. Domain specific challenges associated with automation and scalability are often not addressed in detail. In fact, Chew et al. state that

There is no single set of Verification Validation and Accreditation (VV\&A) tasks, events, or methods that applies exclusively every time to every situation [83].

Even so, this section aims to highlight general aspects needed to make the most of available measurement data in terms of model validation and, in the end, to contribute to simulator credibility assessment. Extensive validation efforts conducted on a high fidelity ECS model of the Gripen Fighter A/C, see Figure 2.1, resulted in a semi-automatic validation framework in Paper [V]. 
This framework is refined here, incorporating the perspective introduced in Paper [I] and Paper [II]. An overview is presented in Figure 5.2. The dashed section of the figure constitute activities initially conducted well in advance of any system level validation activities. However, as model development tends to be iterative (requirements etc. may not be known beforehand) these activities need to be re-visited throughout the model's life-cycle. Here, the Model Specification coincides with the description provided in Section 2.3.

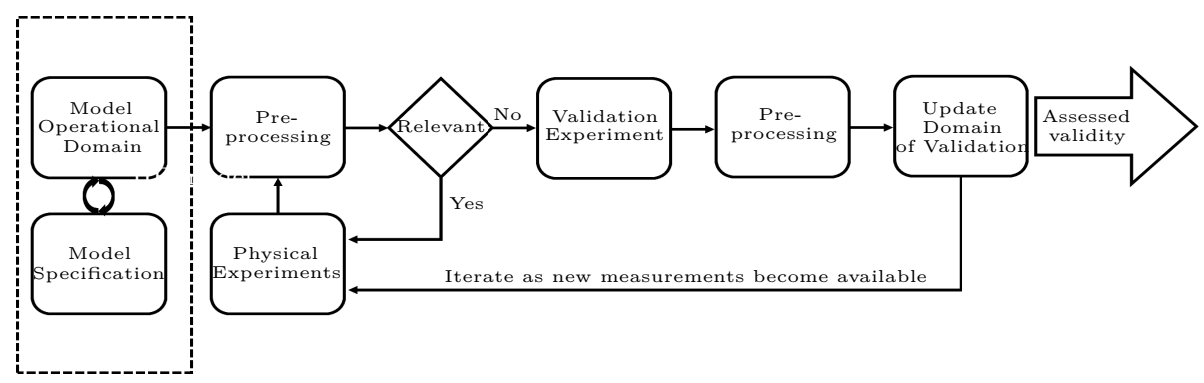

Figure 5.2 Schematic overview of framework for automated model validation.

\subsubsection{Pre-processing}

The pre-processing activity illustrated in Figure 5.2 includes any necessary filtering and re-sampling, categorization of the data, steady-state identification and generation of simulation boundary conditions. The gathered measurement data may be sampled at various rates, and simulation models often require uniform input resolution. In addition, it is often not efficient to feed a model with high resolution data if the model is not meant to represent the corresponding high frequency dynamics of the SoI. The simulation execution is likely to take longer without any gain in information. If the data is to be down-sampled, filtering may be necessary to avoid distorting the measurements as a result of aliasing [60] of present noise and SoI dynamics outside of the model scope of representation.

Categorizing the data is included as a step in this framework as it is important to ensure traceability and efficient CM. For example, storing information regarding the testing station (if there are several stations capable of producing measurements of the same sub-system) may be essential when creating model boundary conditions for conducting validation experiments because the measurement signal names may be different. Steady-state identification is determined as necessary for expressing the steady-state DoV. Various steadystate identification techniques are evaluated in Paper [II]. Comparing the 
DoV expansion occurring as a result of additional validation experiments is one approach to assessing the potential increase in knowledge provided by the physical experiments conducted. Figure 5.3 illustrates how the results of a coverage function $\mathrm{f}_{\mathrm{c}}$ can be used to aid in determining whether to proceed with a particular validation experiment or not. Such functionality is useful if the time available to conduct validation experiments is limited. If so, the knowledge gained from each experiment is of the essence. The nearest neighbor coverage metric, $\eta_{c}$, is one example, see Equation 5.1, of such a coverage function, $f_{c}$. A significant increase in coverage may not be the only condition to consider when establishing whether it is relevant to proceed with conducting the corresponding validation experiments. The SoI may, for example, have been operated outside of the model OD, see Figure 5.1.

So far, the research conducted has been limited to methods establishing steadystate DoVs. In order to similarly quantify the validity of a model's dynamics, methods to express ODs that include transients need to be established. One possibility is to incorporate the time derivatives of the model inputs in the OD. Dimensionality reduction techniques, see for example Andersson et al. for a similar application [84], can then be applied to obtain ODs of manageable dimension. Investigating the feasibility of such an approach is left for future research.

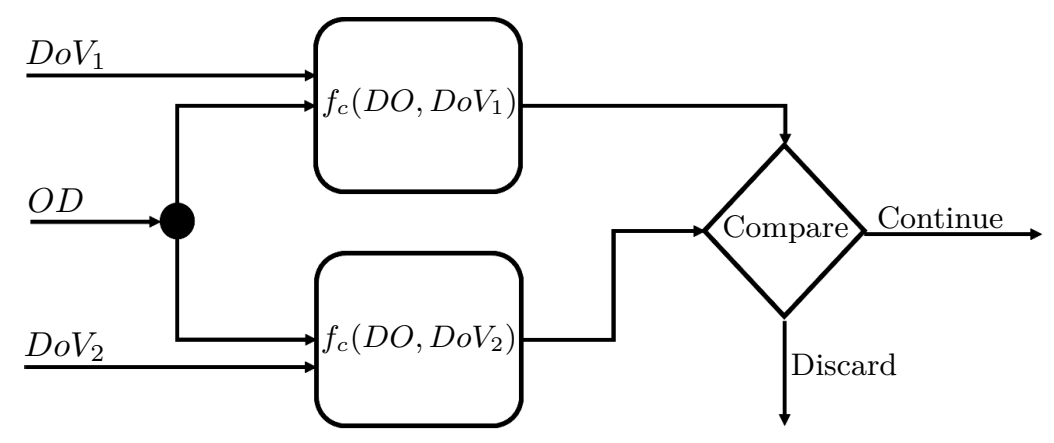

Figure 5.3 Example of how comparisons of the current (DoV $V_{1}$ and a future Domain of Validation $\left(\mathrm{DoV}_{2}\right)$ to the model $\mathrm{OD}$ can be used as criteria for conducting validation experiments. DoV $V_{2}$ represents the DoV, including the validation experiment settings, that is up for evaluation.

The modified nearest neighbor metric is used to exemplify how coverage can be used to quantify the added value of future validation experiments. Figure 5.4 depicts a gridded symmetric OD. The modified nearest neighbor coverage metric is computed in an exhaustive search where, 1) each grid point is selected as the first and only point in the DoV, 2) a second set of validation settings 


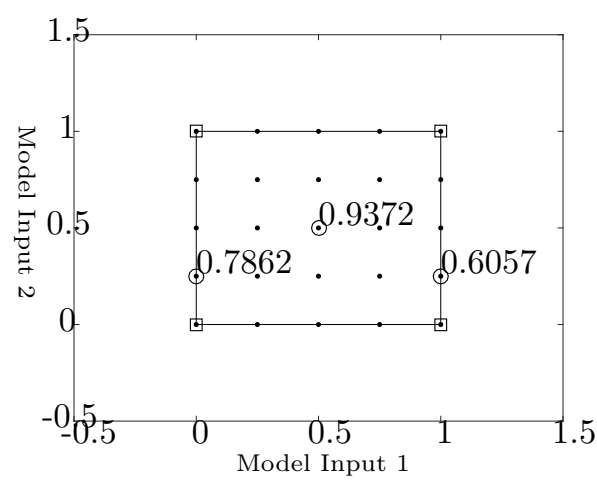

Figure 5.4 Convex hull enclosing a gridded OD. Values of computed nearest neighbor coverage are presented for the first 3 selected experiment settings. The highest value represents the first selected set of model input settings, marked as green in Table 5.1. The computations corresponding to the second and third selected settings are presented in Table C.1 and Table C.2 respectively.
Validation experiment 1

\begin{tabular}{lll}
\hline Model Input 2 & Model Input 1 & $\eta_{c}$ \\
\hline 0 & 0 & 1,5868 \\
0,25 & 0 & 1,3605 \\
0,5 & 0 & 1,2831 \\
0,75 & 0 & 1,3605 \\
1 & 0 & 1,5868 \\
0 & 0,25 & 1,3605 \\
0,25 & 0,25 & 1,1106 \\
0,5 & 0,25 & 1,0253 \\
0,75 & 0,25 & 1,1106 \\
1 & 0,25 & 1,3605 \\
0 & 0,5 & 1,2831 \\
0,25 & 0,5 & 1,0253 \\
0,5 & 0,5 & 0,9372
\end{tabular}

Table 5.1 Selection of initial validation settings, using nearest neighbor coverage, within a rectangular and symmetrical operational domain spanned by two model inputs. The row marked in green represents the optimal first selection of validation settings whereas the rows marked in red represent the worst selections of validation settings. Only half of the data is show in the table as this example is symmetric.

are identified provided the first point is selected optimally, and 3) a third set of validation settings are identified provided the first and second point are successively selected such that $\eta_{c}$ is minimized at every step. The coverage values of all possible selections of initial point are presented in Table 5.1. The corresponding exhaustive search values for the second and third points are presented in Appendix C.

Provided that the measurements are established as relevant for model validation, the model may have to be configured to represent the SoI prior to simulation. It is not unusual for models to be parameterized to represent several different configurations of the same SoI in order to exploit the benefits of reuse. Once configured, the model boundary conditions are input into the model and a validation simulation is conducted. The simulation results are placed under CM and made available for post processing and future analysis. 


\subsubsection{Post-processing}

The post processing phase includes computing relevant validation metrics at both the signal and sub-system levels. In addition, computed validation measures and updated DoVs need to be stored and placed under CM along with the simulation results and the generated model or simulator boundary conditions. As a result, all validation experiments are repeatable and traceable and the history of sub-system validation is available to both developers and end users.

Atamturktur et al. [79] expand the coverage metric to incorporate uncertainties. Similarly, coverage can be modified to include different identified model quality aspects. Forss [85] illustrates how Equation 5.1 can be modified to include a sub-system level validation measure $V_{E}$,

$$
\eta_{c, V}=\frac{1}{g} \sum_{i=1}^{g} \min \left(d_{E, i}\right)\left(1+V_{E}\right)+d_{Z I, i} .
$$

In Equation 5.2, the validation measure $V_{E}$ is used to scale the minimal distance $d_{E, i}$. As a result, it is ensured that a validation experiment indicating a poor model fit will influence $\eta_{c, V}$ for all grid points $i$ in the OD.

Many signal level validation measures exist, and which of these is favorable depends on the engineering domain and application. In the field of system identification, predictive models are estimated from available measurement data. Predictive models aim to predict system outputs, and as such it is often reasonable to minimize a prediction error $\epsilon_{t}(\theta)$ via a loss function $l$ according to

$$
\hat{\theta}=\arg \min _{\theta} \frac{1}{n} \sum_{t=1}^{n} l\left(\epsilon_{t}(\theta)\right) .
$$

This is known as a Prediction Error Method (PEM) and it is used to estimate the optimal model parameter vector $\hat{\theta}$. A typical selection of the loss function is $l\left(\epsilon_{t}\right)=\epsilon_{t}^{2}$ where $\epsilon_{t}$ is the prediction error at time $t$, resulting in the minimization of the mean square prediction error over the $n$ available samples [46, 22]. The mean square prediction error is one signal level measure that is feasible for automated model validation. Oberkampf and Roy [75], as well as Dowding et al. [86], provide several examples of validation efforts where similar measures, based on for example the $\mathrm{L}_{1}$ and $\mathrm{L}_{2}$ vector norms of the prediction error, have been used.

The selected appropriate signal level validation metrics needs to be aggregated to form a system level metric if they are to be incorporated in Equation 5.2. One simple, yet intuitive, selection of system level validation metric is to simply extract the maximum signal level metric value of all model SRQs of interest. The framework presented in Paper [V] incorporates the weighted average of 
all relative errors. This weighted average is designed to act as a type of filter tolerating small relative errors but penalizing large relative errors exponentially.

\subsection{Automating Simulator Credibility Assessment}

In Paper [I], a method for assessing and presenting the credibility of largescale simulators is presented. The method includes equipping each sub-system model with a meta-model. This meta-model is specified as describing the sub-system model credibility by means of dynamic measures of credibility at both the model and signal levels. In addition, inspired by [76] and [87, 88], a set of static measures is presented in Paper [I]. Simulator overall credibility is assessed by means of aggregating the credibility information of each included sub-system model.

The designed meta-model outputs credibility at a higher level of abstraction than what is provided by the system level validation metrics discussed in Section 5.2.2. This is achieved by means of Subject Matter Experts (SMEs) providing subjective input. The SMEs translate the assessed model credibility, via an agreed upon mapping, into a meta-model. The meta-model then provides an integer output describing discrete predefined levels of credibility. Another approach is to first agree upon a generic meta-model with a mathematically defined translation of sub-system model credibility information. Such an approach favors objective V\&V, reuse, and automation. However, it is a challenge to translate the modelers and end users subjective experience on model quality into mathematical expressions.

Once formulated, the meta-model is automatically populated with updated information on assessed model credibility, supporting and enhancing simulator level credibility assessment according to [I], by means of the Validation framework shown in Figure 5.2. A schematic description of this approach is provided in Figure 5.5.

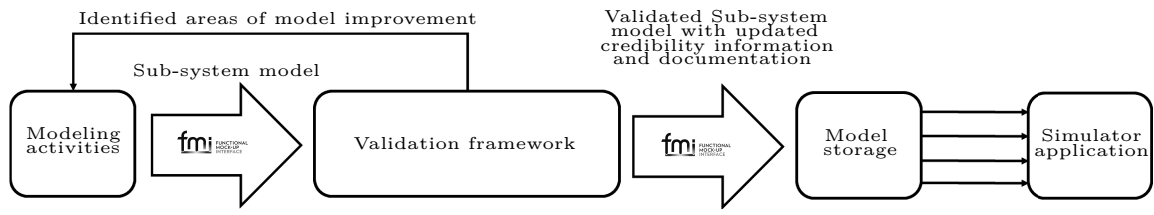

Figure 5.5 Schematic description of proposed flow for development, maintenance, and automated continuous credibility assessment of simulators.

The proposed method is described in the enumerated list below. 
1) A sub-system model is exported from the modeling tool in which it is developed using the standardized FMI format.

2) The model is integrated in the $\mathrm{V} \& \mathrm{~V}$ framework where validation experiments are conducted once the corresponding measurements are available. Validation metrics are computed and the model DoV is updated. The sub-system modeler is notified if non-acceptable discrepancies are detected which may require model updates. The meta-model is updated with the most current credibility information. The model is then placed under $\mathrm{CM}$ in a central model storage.

3) Models are checked out from the central model storage and integrated into relevant small-scale, mid-scale, and large-scale simulator applications.

4) The meta-models of all sub-system models in the simulator application are set to point to the most recent stored assessed credibility information provided that the model placed in the validation framework remains unchanged. Once an updated version of the model is imported in the validation framework, the assessed credibility of the previous model is frozen because it will no longer be updated. That way, the most recent dynamic credibility metric is always presented to the simulator end user.

The presented method is also feasible on a simulator level, supporting the sought after bottom up approach of $V \& V$ presented in Figure 2.3. In that case, a simulator application is integrated into the $\mathrm{V} \& \mathrm{~V}$ framework. The simulator application needs to aggregate the outputs of the constituent sub-system metamodels into a simulator level meta-model. This top level meta-model will then describe the credibility of the simulator application in question. A simple example of a simulator level meta-model is an operator extracting the minimum credibility level provided by the constituent sub-system meta-models. 


\section{6 \\ Discussion and Conclusions}

\subsection{Discussion}

As stated in Chapter 1, in order to fully retain the value of investments in A/C simulation applications, flexible and sustainable methods for model and simulator development, reuse, maintenance, documentation, and V\&V are required. All these aspects are essential, and any major deficiencies will result in sub-optimal product development and operation. The author believes that the topic of model and simulator credibility assessment is of particular interest because its influence on product development is somewhat implicit. For example, model-based decisions with respect to a simulator's intended use can be taken once the simulator is available. However, if there are no investigations of the credibility of the simulator or its constituent models, then there is no telling how accurate these decisions are. In addition, the ambition to increase the use of $\mathrm{M} \& \mathrm{~S}$ during $\mathrm{A} / \mathrm{C}$ development imposes significant requirements on techniques for exchanging, connecting, and jointly simulating executable models. If the credibility assessed at the model level is to say anything about the simulator's credibility, the assembly procedure cannot introduce unknown discrepancies with respect to the SoI.

The vast number of available sensors in modern aircraft is opening up a new world of possibilities for model validation during later development phases. Even so, to be able to make use of all the available information, advances need to be made in automating the model validation processes. As a result, techniques to express model and simulator credibility mathematically is a must. The techniques used need to be scalable and generic such that they are applicable to most sub-system models. Scalable and generic methods not only simplify the bottom up approach to credibility assessment of simulator applications, but 
they are also essential for managing the maintenance and development costs of the validation framework itself.

The importance of Independent Verification and Validation (IV\&V) is stressed in the literature, see for example Gupta [89] and Arthur et al. [90]. In IV\&V, a separate organization responsible for $\mathrm{V} \& \mathrm{~V}$ activities is suggested; primarily in order to achieve objectivity, added analytical perspective, and additional informal testing using independent tools [91]. It is the authors belief that a significant amount of the identified benefits of IV\&V are obtained for "free" when automating validation according to the proposal presented in this thesis. An automated validation framework is seen here as a separate organization capable of computing and evaluating mathematically defined metrics at the same rate as measurements become available.

Even though full independence may be difficult to achieve without humans in the V\&V loop, the use of open standards and tools allows for validation using independent software without increasing the complexity of implementation. Another stipulated benefit of automated validation is that engineers will be freed from tedious and error prone V\&V activities; allowing them to focus on engineering work with, for example, direct impact on the SoI development. However, automating model validation is not deemed as risk free. One challenging aspect is that automated model validation requires the simulation application to be used as originally intended at all times. This is often not the case in practice. If the validation metrics are miss-representative of the actual intended use, the impact on the validation results need to be established manually by humans in the loop. That being said, the end-user may not be aware of this discrepancy resulting in hidden risks that may impact simulation based decisions. Similar problems arise when using manually validated simulation applications; particularly in situations where the end-users are different from the personnel conducting the V\&V. Even so, a manual framework does not produce results without naturally occurring points of bench-marking. The chances of identifying miss-representative intended uses are therefore greater.

One of the contributions of this thesis is the industrial context provided for the developed, investigated, and evaluated standards and techniques for the integration of simulation models. Applying the SotA in academia to existing industrially relevant methods for model based aircraft sub-system development has resulted in more needs than those that have been addressed in this thesis. A sub set of research questions that need to be answered by future research are presented in Section 6.3. Even though the remaining challenges are numerous, automated model and simulator validation, considering aspects such as independence and objectivity, is a realistic future achievement. 


\subsection{Conclusions}

The research questions first presented in Chapter 1 are re-stated below in order to remind the reader prior to reflecting upon the answers presented in this section.

\section{RQ1: Is the $F M I$ standard applicable for the aeronautical industry and A/C vehicle system development?}

A scalable mid-scale A/C simulator is developed in papers [VI, IV, VII]. The simulator is, among other things, intended to answer RQ1 demonstrating FMI as a feasible option for the standardized exchange of $\mathrm{A} / \mathrm{C}$ sub-system simulation models. The feasibility of FMI is shown by means of conducting an industry relevant study connecting ECS performance to pilot thermal comfort. Models from the engineering domains of $\mathrm{H} / \mathrm{W}, \mathrm{S} / \mathrm{W}$, and human factors are successfully incorporated into the simulator exemplifying the interoperability established via FMI. Finally, a mission profile is simulated in the open source OpenCPS effort the OMSimulator.

\section{RQ2: Can multiple connected $A / C$ sub-system be simulated in parallel without affecting accuracy and numerical stability?}

A second purpose of the mid-scale simulator mentioned above is to demonstrate interoperability between TLM and FMI in order to achieve numerically robust and parallel co-simulation. However, early development of this simulator, see Paper [VI], revealed that the approach of establishing aforementioned interoperability via sub-stepping is insufficient in terms of numerical stability. Consequently, a method for passing high resolution interpolation data with the current version of FMI is developed in Paper [III]. This approach is successfully evaluated in Paper [IV] and Paper [VII] where results deemed as accurate were obtained via a parallel simulation. The TLM approach was compared to a corresponding sequential simulator implementation in Paper [VIII].

Furthermore, Paper [IV] and Paper [III] contributed to an accepted FMI standard change proposal allowing for access to input and output information at the discretion of each incorporated FMU. As identified by Benedikt et al. [16], the use of TLM-type connections requires modeling-side modification, particularly if considering the fine-grained approach compatible with FMI 2.0. This drawback is significantly reduced under the accepted FMI proposal; however, modifications are still necessary, see Chapter 4. Modifying models to accommodate TLM type connections is not always a possibility. In such a case, it may very well be better to rely on knowledge concerning the system dynamic 
properties to ensure numerical accuracy and stability.

\section{RQ3: What aspects need to be addressed if $V \& V$ in later development phases is to be automated?}

The mid-scale simulator of Paper [IV] was implemented in the OMSimulator. This mid-scale simulator was used to demonstrate how models from various domains can be assembled and simulated in an open-source tool on an industrially relevant scale. In addition, the SSP standard was investigated and the simulator was used to demonstrate the exchange of simulation architectures between the tools OMSimulator and Papyrus, see Section 3.3. In answer to RQ3, the OMSimulator functionality provides a key piece of the puzzle to achieving an industrially relevant framework for automated model validation; an open-source tool able to numerically accurately simulate coupled aircraft vehicle system models using open standards for information exchange. Automated model validation is here partitioned into two different parts separated by the definitions of ODs: model validation during steady-state and transient operation respectively. The topic of steady-state operation is addressed in Paper [II] in which available methods for steady-state identification were investigated and applied to flight measurements. Once steady-state operation is identified, comparisons between ODs and DoVs can be made to compute coverage and system level validation metrics, see Chapter 5 . If it were possible to express ODs including identified dynamic phenomena, similar coverage and validation metrics could be applied to account for the accuracy of modeled dynamics. One possible solution could be to include time derivatives of the inputs to the OD and use dimension reduction techniques, see Andersson et al. [84], to obtain ODs of manageable dimension.

Here, the nearest neighbor coverage metric is used to account for coverage, see Equation 5.1, and as a system level validation metric, see Equation 5.2. The nearest neighbour coverage metric requires a gridded OD, which is a significant computational challenge for models with input space of large dimension. For such models, a different approach may be necessary.

\section{RQ4: How can the validity of $A / C$ sub-systems be propagated to simulator level?}

In answer to RQ4, a method for assessing and presenting the credibility of simulators, directly during simulation, is proposed and evaluated in Paper [I]. Each model included in a simulator is appended with a meta-model describing different aspects of sub-system model credibility. This meta-model is populated with information concerning validity originating from the normal V\&V procedures [7] of Saab Aeronautics. The outcomes of Paper [I] and Paper [II] 
are used to refine the framework proposed in Paper [V] for model validation. The outline of the refined framework is presented in Chapter 5 where the results from automated model validation are proposed to populate sub-system meta-models for on-line simulator credibility assessment.

There is a fine balance between keeping metrics conveying credibility simple enough to be understandable to end-users while complex enough to actually say something about the model's credibility. The meta-model solution does help in addressing this challenge. A meta-model example with a proposed standardized interface is provided in Paper [I]. Such a meta-model can act as a parser of complex metrics to provide coarse but relevant information about model and, in the end, simulator credibility.

\subsection{Outlook and Future Work}

Efficient model integration is identified as a key area for future research by Saab and the European M\&S community at large, with accepted research initiatives such as the NFFP 7 research project Digital Twin for Automated Flight Test Evaluation and Model Validation [92], and the ITEA 3 project Environment for model-based rigorous adaptive co-design and operations of $C P S(E M B r A C E)$ [93].

The research presented here has been received by the Saab Aeronautics key technology area Compact and Efficient Platform in which the Technology Readiness Level (TRL) of the results will be successively increased. The ambition is to have an implemented and operational framework for automated model validation and anomaly detection in place by the time the next large development project commences.

Implementing a framework as described in Chapter 5 does not only increase the confidence in M\&S method and applications. The applications of automated and model-based anomaly detection and prognosis emerge as feasible once the models and simulators credibility with respect to the SoI is established. Another possible spin-off is the improved planning of flight tests conducted with the specific purpose of validating models and simulators. This topic is touched upon in Chapter 5. Further improvements to the presented approach are to use model in the loop optimization to maximize knowledge gained with respect to, for example, consumed fuel. A number of research questions have emerged as a result of the presented work. A selected few of particular interest are presented in the list below. These questions, among others, will be investigated in the accepted NFFP 7 research project mentioned above. 
1) How can techniques for model validation during steady-state conditions be adapted to assess the transient operation validity?

2) How can metrics of operational domain coverage be used to reduce the number of required validation experiments and flight tests?

3) How can model in the loop optimization reduce the costs of assessing model and simulator credibility? 


\section{A \\ FMI and SSP Examples}

\section{A.1 Exchange of Models}

The example provided in Figure 3.1 is implemented in Dymola. This particular example is constructed in order to visualize the most fundamental aspects relevant for this thesis. The two modeled sub-systems are exported as FMUs and the resulting Subsystem A ModelDescription file is presented in Listings A.1.

The ModelDescription file presented in Listings A.1 shows that this particular FMU contains binaries for both ME and CS. This feature is not a requirement but an export option that is mentioned in the standard specification and is available in Dymola. If the optional capability flag providesDirectionalDerivative, seen at the top of Listings A.1, is specified as true, then the FMU directional derivatives can be obtained using the specified function fmi2GetDirectionalDerivative. The capability flag canGetAndSetFMUState states whether the FMU supports the functions fmi2GetFMUstate, fmi2SetFMUstate, and fmi2FreeFMUstate, or not. If these functions are supported, the MST can acquire and set the FMU internal states, allowing for advanced master algorithms supporting, for example, roll back. The last capability flag shown in Listings A.1 is denoted canHandleVariableCommunicationStepSize seen under the CoSimulation element. This capability flag states that the FMU internal solver can handle non-constant step sizes in the fmi2DoStep function; the flag is only relevant for the CS case as the model equations are solved by the MST in the ME case. The modelIdentifier attribute under the ModelExchange and CoSimulation elements states the model name. The UnitDefinitions and TypeDefinitions are not expanded. However, the UnitDefinitions element presents the actual and display units of the variables 
listed under the ModelVariables element. The TypeDefinitions element is used to define all the types used in the ModelVariables via subordinate SimpleType elements. The simple type elements contain the attributes name and type. The types allowed in FMI 2.0 are Real, Integer, Boolean, String, and Enumeration.

The previously mentioned ModelVariables element includes a list of all accessible variables in the model. Each variable is listed as a ScalarVariable element assigned with one of the previously defined types and a number of attributes. Examples of ScalarVariable attributes are name, valueReference, causality, and variability. The valueReference is a unique number assigned to each model variable. The example valueReference value has been omitted from the listings. This number is used by the MST to reference the specific variable. If the variable is an input, output or parameter is stated via the causality attribute and the variability attribute states how the variable is allowed to change, or not, throughout the simulation.

The ModelStructure element states the structure of the exported model. This information can be used to, for example, identify algebraic loops. If focusing on the Outputs element. The xml file first states that the ModelVariable with index $5, y_{A}^{I}$, directly depends on the ModelVariable with index $4 u_{A}^{I}$. This direct dependency could cause an a algebraic loop. The second output $y_{A}^{I I}$ with index 7 does not directly depend on any input. 
Listing A.1 ModelDescription xml file of Subsystem A in Figure 3.1. Nonrelevant information, such as the FMU value reference numbers have been removed from the xml code. All ScalarVariables of causality different from "input" and "output" have been removed to simplify readability.

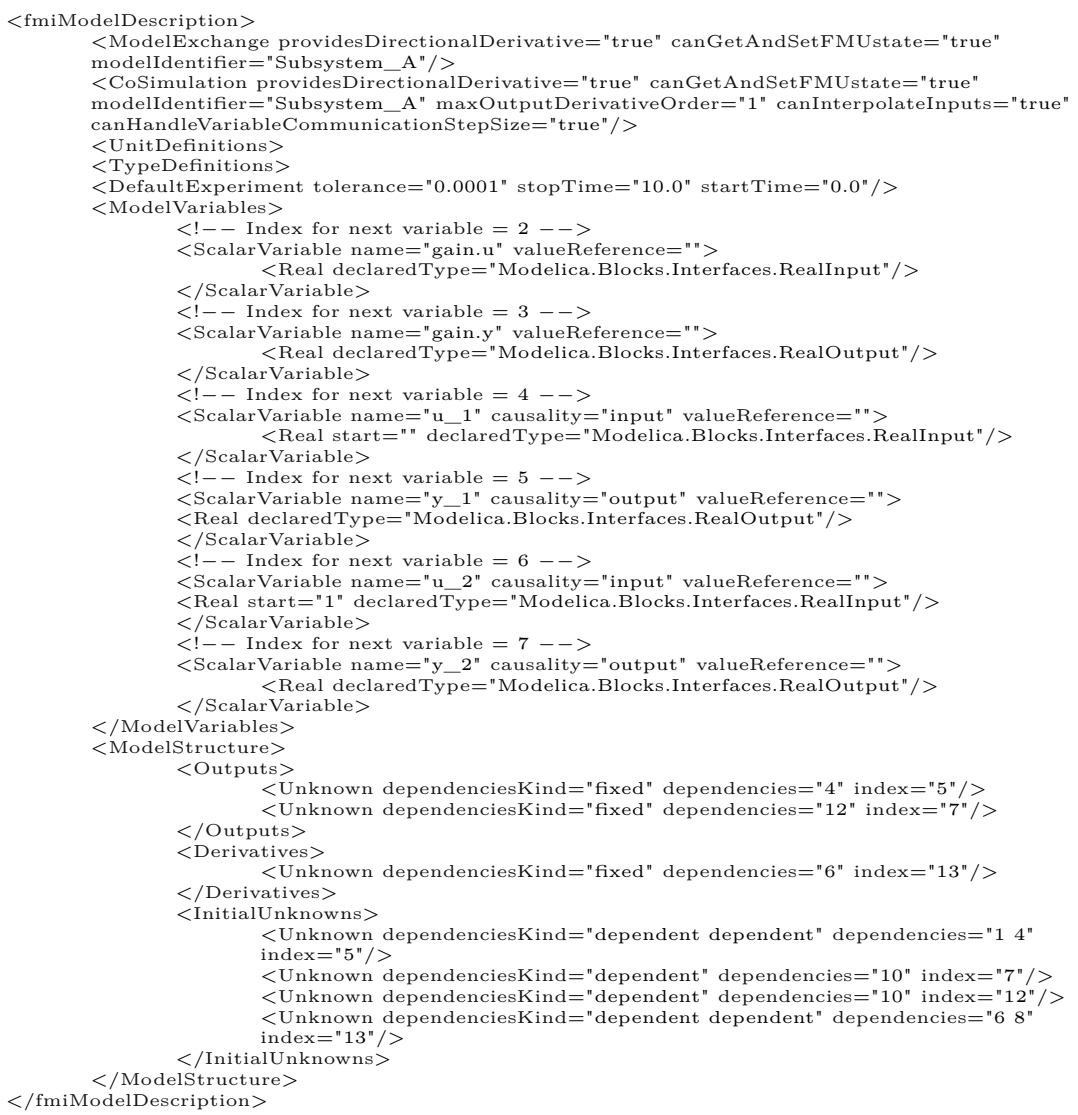




\section{A.2 Simulator Exchange}

The models of the example provided in Figure 3.1 are implemented in Dymola. The two modeled sub-systems are exported as FMUs. The FMUs are imported into OMEdit and the connections are established according to the example specification. The resulting simulator is exported as an SSP file where the architecture is described by the comprising SSD xml file. The latter is provided in Listings A.2. The example SSD file is provided as a basis for explaining the fundamental properties of the SSP standard [35]. Text marked as cursive in the description below indicate xml elements or attributes of the schema.

The first xml element, System, of Listings A.2, describes the top level of the simulator architecture. The System of the example is given name "Root" via the name attribute of the element. The System has children Elements and Connections.

The child Elements incorporates the included Components specified as the FMUs "SubsystemB.fmu" and "SubsystemA.fmu". Each Component has the attributes name, type, and source. The attribute name sets the name of the Component. The type attribute indicates whether the component is an FMU, an SSP package, or another SSD file. These three types are the ones specified in the standard specification. However, a future possibility would be to support a type considering direct connection to an M\&S tool. The source attribute specifies the path to the resources of the component, in the example, the corresponding FMU.

A Component can have several child elements according to the specification: Connectors, ElementGeometry, and ParameterBindings. The Connectors element represents the interface of incorporated model and is, in the case of the example, mapped to the interface information of each FMUs ModelDescription file. A Connector element has attributes name, kind, and type. These attributes must match the ScalarVariable attributes name and causality of the model description file. The SSD attribute kind must match the type of the considered ScalarVariable specified in the TypeDefinition element of the ModelDescription file. The ElementGeometry element is optional and it describes geometry information of the component. The ParameterBindings element specifies the application of a set of parameters applicable for the component that are supplied from, for example, a specified source file. This ParameterBindings is optional and not included in the example.

The second visualized System child Connections specifies the top level simulator connections. The Connections element has children denoted Connection representing the individual couplings between Connectors. The Connector attributes startElement and endElement give the names of the elements contain- 


\section{ing the startConnector and endConnector attributes. The latter two attributes are required and need to map to corresponding Component Connectors.}

\section{Listing A.2 SSD xml file describing the simulator architecture presented in Figure 3.1.}

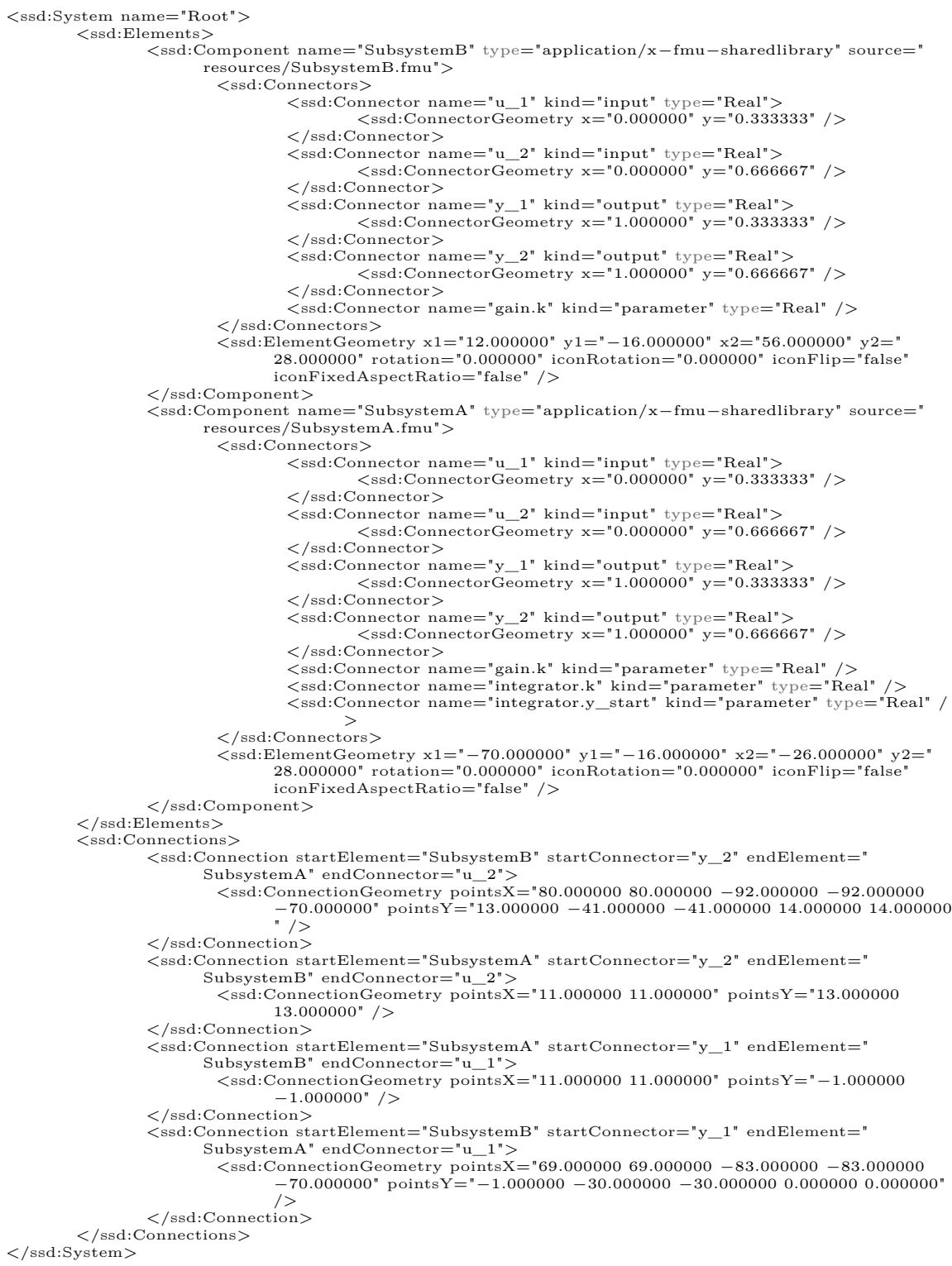




\section{B \\ Derivation of TLM Equations}

The equations describing a one dimensional, loss-less, hydraulic transmission line are derived in this section. Such a transmission line is schematically visualized in Figure B.1.

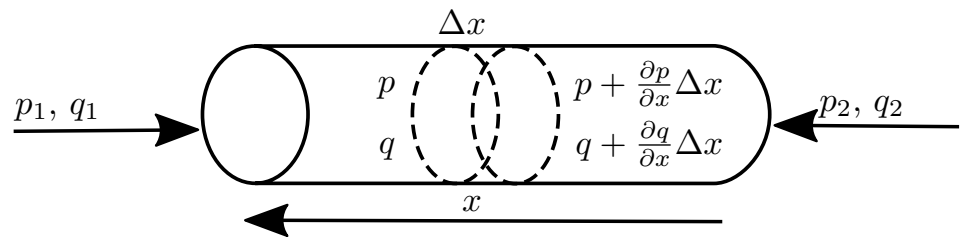

Figure B.1 One dimensional, loss-less, hydraulic transmission line modeled as a pipe with length $l_{\text {pipe }}$. Each side of the element is input a pressure and a volumetric flow.

Newton's second law in one dimension for a fluid element with length $\Delta x$, and cross sectional area $A$, is

$$
A \Delta x \frac{\partial p}{\partial x}=\rho A \Delta x \frac{\partial v}{\partial t}
$$

where the left hand side of the equation is the net force acting on the fluid element. The total mass of the element is represented by $\rho A \Delta x$. The characteristic acoustic impedance is defined as

$$
Z_{c}=\frac{\rho a}{A}
$$

for a one dimensional acoustic wave. The speed of sound is denoted $a$ in 
Equation B.2. Inserting Equation B.2 into Equation B.1 yields

$$
\frac{\partial p(t, x)}{\partial x}=\frac{Z_{c}}{a} \frac{\partial q(t, x)}{\partial t}
$$

after some simplifications. Equation B.3 is known as one of the two telegraphers equations. The second telegraphers equation is here derived from,

$$
\sum q_{i}=\frac{d V}{d t}+\frac{V}{\beta} \frac{\partial p}{\partial t}
$$

the continuity equation [94]. The volume $V=A \Delta x$ of the fluid element in Figure B.1 does not change with time, Equation B.4 can therefore be re-written as

$$
\frac{\partial q}{\partial x} \Delta x=\frac{A \Delta x}{\beta} \frac{\partial p}{\partial t}
$$

and if the the Newton-Laplace equation

$$
a=\sqrt{\beta / \rho}
$$

is inserted into Equation B.5 along with Equation B.2; then the second telegraphers equation

$$
\frac{\partial p(t, x)}{\partial t}=a Z_{c} \frac{\partial q(t, x)}{\partial x}
$$

is obtained. Differentiating Equation B.3 and Equation B.7 with respect to $t$ and $x$ respectively results in

$$
\frac{\partial^{2} q(t, x)}{\partial t^{2}}=a^{2} \frac{\partial^{2} q(t, x)}{\partial x^{2}}
$$

which is known as the wave-equation. The general solution to the wave-equation in the Laplace domain,

$$
Q(s, x)=A_{1} e^{\frac{s x}{a}}+B_{1} e^{-\frac{s x}{a}},
$$

can be found by means of taking the Laplace transform of Equation B.8 and applying the initial conditions $q(0, x)=0$ and $\frac{\partial q}{\partial t}(0, x)=0$. Inserting the solution, along with the initial condition $p(0, x)=0$, into the Laplace transform of Equation B.7 yields

$$
P(s, x)=Z_{c}\left(A_{1} e^{\frac{s x}{a}}-B_{1} e^{-\frac{s x}{a}}\right)
$$

after some straightforward simplifications. The boundary conditions, see Figure B.1, are

$$
\begin{aligned}
P(s, 0) & =P_{1}(s) \\
Q(s, 0) & =-Q_{1}(s) \\
P\left(s, a \Delta t_{T L M}\right) & =P_{2}(s) \\
Q\left(s, a \Delta t_{T L M}\right) & =Q_{2}(s)
\end{aligned}
$$


in the Laplace domain. The constants $A$ and $B$ are determined using the boundary conditions. Once the constants are known, Equation B.9 and Equation B.10 can be re-written as

$$
Q(s, x)=\frac{P_{1}(s)}{Z_{c}}\left(\frac{e^{\frac{s x}{a}}-e^{\frac{-s x}{a}}}{2}\right)-Q_{1}(s)\left(\frac{e^{\frac{-s x}{a}}+e^{\frac{-s x}{a}}}{2}\right)
$$

and

$$
P(s, x)=P_{1}(s)\left(\frac{e^{\frac{s x}{a}}+e^{\frac{-s x}{a}}}{2}\right)-Z_{c} Q_{1}(s)\left(\frac{e^{\frac{s x}{a}}-e^{\frac{-s x}{a}}}{2}\right)
$$

respectively. Subtracting Equation B.12 multiplied by $Z_{c}$ from Equation B.13 yields

$$
P(s, x)-Z_{c} Q(s, x)=\left(P_{1}(s)+Z_{c} Q_{1}(s)\right) e^{\frac{-s x}{a}} .
$$

Focusing on the rightmost end of the transmission line element, $x=a \Delta t_{T L M}$ and taking the inverse Laplace transform of Equation B.14 gives

$$
p_{2}(t)-Z_{c} q_{2}(t)=p_{1}\left(t-\Delta t_{T L M}\right)+Z_{c} q_{2}\left(t-\Delta t_{T L M}\right)
$$

the first of the time domain TLM equations. The second equation is presented in Equation B.16,

$$
p_{1}(t)-Z_{c} q_{1}(t)=p_{2}\left(t-\Delta t_{T L M}\right)+Z_{c} q_{2}\left(t-\Delta t_{T L M}\right) .
$$

and it can be derived analogously or simply stated as a result of symmetry. 


\section{C Validation Experiments}

The second and third proposed validation experiment settings presented in Figure 5.4 are derived using the nearest neighbor coverage, see Equation 5.1, computations presented in Table C.1 and Table C.2. 


\section{C.1 Experiment 2}

During the second iteration, selecting point $(0.50 .5)$ as the next set of validation experiments would not increase knowledge on coverage as these settings already have been tested, see Table 5.1 , a property that is clearly revealed in Table C.1. In Table C.1, $\sum_{i=1}^{g} d_{E, i}$ and $\sum_{i=1}^{g} d_{Z I, i}$ are different from one and another. In this phase, the distance $d_{Z I, i}$ is the distance from grid point $i$ to the line in between the two selected sets whereas, in the first iteration of Table 5.1 , it is the distance from grid point $i$ to a single selected point.

Validation Experiment 2

\begin{tabular}{lllll}
\hline Model Input 2 & Model Input 1 & $\sum_{i=1}^{g} \min \left(d_{E, i}\right)$ & $\sum_{i=1}^{g} d_{Z I, i}$ & $\eta_{c}$ \\
\hline 0 & 0 & 10,3896 & 9,3929 & 0,7913 \\
0,25 & 0 & 10,0806 & 9,5733 & 0,7862 \\
0,5 & 0 & 10,1825 & 9,6074 & 0,7916 \\
0,75 & 0 & 10,0806 & 9,5733 & 0,7862 \\
1 & 0 & 10,3896 & 9,3929 & 0,7913 \\
0 & 0,25 & 10,0806 & 9,5733 & 0,7862 \\
0,25 & 0,25 & 10,0967 & 9,8929 & 0,7996 \\
0,5 & 0,25 & 10,1825 & 10,1825 & 0,8146 \\
0,75 & 0,25 & 10,0967 & 9,8929 & 0,7996 \\
1 & 0,25 & 10,0806 & 9,5733 & 0,7862 \\
0 & 0,5 & 10,1825 & 9,6074 & 0,7916 \\
0,25 & 0,5 & 10,1825 & 10,1825 & 0,8146 \\
0,5 & 0,5 & 11,7148 & 11,7148 & 0,9372
\end{tabular}

Table C.1 Selection of second set of validation settings given that the first set was selected optimally with respect to the nearest neighbour coverage metric. The rows marked as green corresponds represents the optimal selection of validation settings. The row marked as red was selected first, see Table 5.1, and does therefore not contribute with new information. Only half of the data is show in the table as this example is symmetric

\section{C.2 Experiment 3}

In Table C.2, any of the two previously chosen sets are depicted as the least favorable third set selection, see the rows marked as red. Equation 5.1 favors diversity in validation experiments. The selected settings of (1.00 0.25) encloses a total of six grid points within the resulting convex hull, meaning that a total of six $d_{Z I, i}$ are zero. In Table C.2, the settings $(0.751)$ have the same coverage metric value as the settings of $(1.000 .25)$. However, this is a result of round off and the point is in fact a worse selection as the extrapolation penalty is 
slightly larger.

Validation Experiment 3

\begin{tabular}{lllll}
\hline Model Input 2 & Model Input 1 & $\sum_{i=1}^{g} \min \left(d_{E, i}\right)$ & $\sum_{i=1}^{g} d_{Z I, i}$ & $\eta_{c}$ \\
\hline 0 & 0 & 9,7271 & 8,8837 & 0,7444 \\
0,25 & 0 & 10,0806 & 9,5733 & 0,7862 \\
0,5 & 0 & 9,3735 & 8,7254 & 0,724 \\
0,75 & 0 & 8,8145 & 7,8435 & 0,6663 \\
1 & 0 & 8,8145 & 7,2063 & 0,6408 \\
0 & 0,25 & 9,418 & 8,5512 & 0,7188 \\
0,25 & 0,25 & 9,5806 & 9,2069 & 0,7515 \\
0,5 & 0,25 & 9,3735 & 9,0648 & 0,7375 \\
0,75 & 0,25 & 8,668 & 7,916 & 0,6634 \\
1 & 0,25 & 8,5055 & 7,1701 & 0,6270 \\
0 & 0,5 & 8,9609 & 7,862 & 0,6729 \\
0,25 & 0,5 & 9,0645 & 8,4391 & 0,7001 \\
0,5 & 0,5 & 10,0806 & 9,5733 & 0,7862 \\
0,75 & 0,5 & 8,6074 & 7,8003 & 0,6563 \\
1 & 0,5 & 8,5484 & 6,9243 & 0,6189 \\
0 & 0,75 & 8,5055 & 7,1189 & 0,6270 \\
0,25 & 0,75 & 8,4626 & 7,3990 & 0,6345 \\
0,5 & 0,75 & 8,5484 & 7,8332 & 0,6553 \\
0,75 & 0,75 & 8,4626 & 7,4814 & 0,6378 \\
1 & 0,75 & 8,4465 & 6,6959 & 0,6057 \\
0 & 1 & 8,7555 & 6,5653 & 0,6128 \\
0,25 & 1 & 8,4465 & 6,6957 & 0,6057 \\
0,5 & 1 & 8,5484 & 7,0307 & 0,6232 \\
0,75 & 1 & 8,4465 & 9,5733 & 0,7208 \\
1 & 1 & 8,7555 & 6,7519 & 0,6203
\end{tabular}

Table C.2 Selection of third set of validation settings given that the first two sets were selected optimally with respect to the nearest neighbour coverage metric. The row marked as green corresponds to the optimal selection of validation settings. The rows marked as red were previously selected, see Table 5.1 and Table C.1, and does therefore not contribute with new information. 


\section{Bibliography}

[1] Stewart Robinson, Richard E. Nance, Ray J. Paul, Michael Pidd, and Simon J.E. Taylor. "Simulation model reuse: definitions, benefits and obstacles". In: Simulation Modelling Practice and Theory 12.7-8 (Nov. 2004), pp. 479-494. DOI: doi:10.1016/j .simpat.2003.11.006.

[2] Peter Fritzson. Principles of Object Oriented Modeling and Simulation with Modelica 2.1. Wiley-IEEE Press, Jan. 2004. ISBN: 9780470545669. DOI: $10.1109 / 9780470545669$.

[3] Ingela Lind and Henric Andersson. "Model Based Systems Engineering for Aircraft Systems - How does Modelica Based Tools Fit?" In: Proceedings of the 8th Modelica Conference. Dresden, Germany, 2011.

[4] Sören Steinkellner. "Aircraft Vehicle Systems Modeling and Simulation under Uncertainty". Licentiate thesis. Linköping University, Division of Machine Design, 2011. ISBN: 9789173931366.

[5] Magnus Carlsson. "Methods for Early Model Validation : Applied on Simulation Models of Aircraft Vehicle Systems". Licentiate thesis. Linköping University, Division of Machine Design, 2013. ISBN: 978-917519-627-5.

[6] Magnus Eek. "On Credibility Assessment in Aircraft System Simulation". PhD thesis. Linköping University, Division of Machine Design, 2016. ISBN: 978-91-7685-780-9.

[7] Henric Andersson and Magnus Carlsson. Saab Aeronautics Handbook for Development of Simulation Models : Public Variant. Tech. rep. 12/00159. Linköping University, Machine Design, 2012.

[8] Daniel P. Raymer. Aircraft design: a conceptual approach. 6th ed. American Institute of Aeronautics Astronautics, 2018. ISBN: 9781624104909.

[9] Michael Grieves and John Vickers. "Digital Twin: Mitigating Unpredictable, Undesirable Emergent Behavior in Complex Systems". In: Transdisciplinary Perspectives on Complex Systems. Springer International Publishing, Aug. 2017, pp. 85-113. ISBN: 978-3-319-38754-3. DOI: 10.1007/978-3-319-38756-7_4. 
[10] Brenna Sniderman, Monika Mahto, and Mark J. Cotteleer. Industry 4.0 and manufacturing ecosystems. Exploring the world of connected enterprises. Report. Deloitte, 2016.

[11] Ulrich Dahmen and Juergen Rossmann. "Experimentable Digital Twins for a Modeling and Simulation-based Engineering Approach". In: 2018 IEEE International Systems Engineering Symposium (ISSE). IEEE, Oct. 2018. DOI: 10.1109/SysEng. 2018.8544383.

[12] Giulio Bondani and Gianluca Bacchiega. "Creating an Embedded Digital Twin: monitor, understand and predict Device Health Failure". In: Inn4mech - Mechatronics and Industry 4.0 Conference Presentation. Ingegneria Ricerca Sistemi. Nov. 26, 2019.

[13] OpenCPS project partners. Project 14018:Open Cyber-Physical System Model-Driven Certified Development. URL: https ://www . opencps.eu/ (visited on 11/15/2019).

[14] International Council of Systems Engineering (INCOSE). Systems Engineering Vision 2020. Tech. rep. INCOSE, 2007.

[15] OpenCPS project partners. D2.2: Interoperability of the standards Modelica-UML-FMI including Annex M36. Tech. rep. ITEA3 Project no. 14018, Dec. 4, 2018.

[16] Martin Benedikt and Edo Drenth. "Relaxing Stiff System Integration by Smoothing Techniques for Non-iterative Co-simulation". In: IUTAM Symposium on Solver-Coupling and Co-Simulation. Springer International Publishing, 2019, pp. 1-25. DOI: https://doi.org/10.1007/ 978-3-030-14883-6_1.

[17] C. Potts. "Software-engineering research revisited". In: IEEE Software 10 (Sept. 1993), pp. 19-28.

[18] G. Muller. Industry-as-Laboratory Applied in Practice: The Boderc Project. Oct. 2017. URL: http : //www gaudisite.nl/ (visited on $11 / 15 / 2019)$.

[19] Christina Johansson. "Multiobjective Optimization for Safety and Reliability Trade-off. Applications on Early Phases of Aircraft System Design". PhD thesis. Linköping University, Division of Machine Design, 2017. ISBN: 9789176855768.

[20] OpenCPS project partners. D2.1: FMI Master Simulation Tool Requirement Specification. Tech. rep. ITEA3 Project no. 14018, 2016.

[21] FMI development group. What will be the new features of FMI 3.0. Oct. 31, 2019. URL: https://fmi-standard.org/faq/\#what-willbe-the-new-features-of-fmi-30 (visited on 06/04/2019).

[22] Lennart Ljung and Torkel Glad. Modelbygge och Simulering. Vol. 2. Studentliteratur, 2004. ISBN: 91-44-02443-6. 
[23] National Aeronautics and Space Administration (NASA). NASA System Engineering Handbook. 12th Media Services, Sept. 19, 2017. ISBN: 9781680920895 .

[24] Henric Andersson. "Variability and Customization of Simulator Products : A Product Line Approach in Model Based Systems Engineering". PhD thesis. Linköping University, Machine Design, 2012. ISBN: 978-917519-963-4.

[25] Henric Andersson. "Aircraft Systems Modeling : Model Based Systems Engineering in Avionics Design and Aircraft Simulation". Licentiate thesis. Linköping University, Division of Machine Design, 2009. ISBN: 978-91-7393-692-7.

[26] Henric Andersson, Erik Herzog, and Johan Ölvander. "Experience from Model and Software Reuse in Aircraft Simulator Product Line Engineering". In: Information and Software Technology 55.3 (2013), pp. 595-606. DOI: 10.1016/j.inf sof .2012.06.014.

[27] Gunilla Sivard. "A generic information platform for product families". PhD thesis. KTH, Production Engineering, 2001.

[28] Merriam-Webster. Merriam-Webster, Since 1828, Dictionary. Feb. 19, 2019. URL: https : / / www . merriam-webster . com / dictionary / integrating (visited on 02/19/2019).

[29] International Council on Systems Engineering. Systems Engineering Handbook. 4th ed. John Wiley and Sons, Inc., 2015. ISBN: 9781118999400 .

[30] Magnus Carlsson, Henric Andersson, Hampus Gavel, and Johan Ölvander. "Methodology for Development and Validation of Multipurpose Simulation Models". In: 50th AIAA Aerospace Sciences Meeting including the New Horizons Forum and Aerospace Exposition. American Institute of Aeronautics and Astronautics, Jan. 2012. DOI: https: //doi.org/10.2514/6.2012-877.

[31] J. Shetty, C.P. Lawson, and A.Z. Shahneh. "Simulation for temperature control of a military aircraft cockpit to avoid pilot's thermal stress." In: CEAS Aeronautical Journal 6.2 (2015), pp. 319-333.

[32] Osman Balci. "Verification, validation and accreditation of simulation models". In: Proceedings of the 1997 Winter Simulation Conference. 1997. DOI: 10.1109/WSC.1997.640389.

[33] FMI development group. Functional Mock-up Interface for Model Exchange and Co-Simulation. Report 2.0. July 25, 2015.

[34] FMI development group. FMI Functional Mock-up Interface. Oct. 31, 2019. URL: https://fmi-standard.org/ (visited on 11/15/2019).

[35] Modelica Association. System Structure and Parameterization. Report 1.0. Mar. 5, 2019. 
[36] Modelica Association Project System Structure and Parameterization. System Structure and Parameterization. URL: https : / / sspstandard.org (visited on 10/23/2019).

[37] Jochen Köler, Hans-Maertin Heinkel, Perre Mai, Jürgen Krasser, Markus Deppe, and Mikio Nagasawa. "Modelica-Association-Project "System Structure and Parameterization" - Early Insights". In: Proceedings of the 1st Japanese Modelica Conference. Tokyo, Japan, 2016.

[38] The Modelica Association. Modelica and the Modelica Association. URL: https://www.modelica.org/ (visited on 11/15/2019).

[39] Dassault Systemes AB. Dymola User Manual. 1st ed. Sept. 2016.

[40] Peter Fritzson et al. "The OpenModelica Modeling, Simulation, and Software Development Environment". In: Simulation News Europe 44 (Dec. 2005), pp. 8-16. ISSN: 0929-2268.

[41] MathWorks. MathWorks Documentation. 2018. URL: https : / / se . mathworks . com/help/simulink/ug/work-with-fmi-in-simulink . html (visited on 11/15/2019).

[42] Dassault Systemes AB. FMI Kit for Simulink 2.3.0. Oct. 2016.

[43] Modelon AB. FMI Toolbox User's Guide 2.6.4. July 23, 2018.

[44] R. Kübler and W. Schiehlen. "Two Methods of Simulator Coupling". In: Mathematical and Computer Modelling of Dynamical Systems 6.2 (2000), pp. 93-113. DOI: 10 . 1076/1387-3954(200006) 6:2 ; 1-M ; FT093.

[45] David Broman et al. "Determinate Composition of FMUs for Cosimulation". In: Proceedings of the Eleventh ACM International Conference on Embedded Software. EMSOFT '13. Piscataway, NJ, USA: IEEE Press, 2013.

[46] Lennart Ljung. System identification: theory for the user. 2nd ed. Prentice Hall, 1999. ISBN: 0136566952.

[47] Judith S. Dahmann, Richard M. Fujimoto, and Richard M. Weatherly. "The Department of Defence High Level Architecture". In: Proceedings of the $199^{\text {r }}$ Winter Simulation Conference. ACM Press, 1997. DOI: 10. $1145 / 268437.268465$.

[48] Alberto Falcone, Anastasia Anagnosou, Alfredo Garro, and Simon J. E. Taylor. "An introduction to developing federations with the high level architecture (HLA)". In: Proceedings of the 2017 winter simulation conference. Las Vegas, NV, USA: IEEE Press, 2017.

[49] Björn Möller. The HLA tutorial v1.0. Ed. by Pitch Technologies. 2013.

[50] Nicke Sievert. "Modelica Models in a Distributed Environment Using FMI and HLA". MA thesis. Department of Computer and Information Science, May 29, 2016. 
[51] OMG Unified Modeling Language (OMG UML), Version 2.5. ptc/201309-05. OMG. 2013.

[52] Francis Bordeleau. "Model-Based Engineering: A New Era Based on Papyrus and Open Source Tooling". In: OSS4MDE@MoDELS. 2014.

[53] Modelon. FMI Composer User's Guide 1.0. Nov. 10, 2017.

[54] Claude Lacoursière and Tomas Härdin. "FMI Go! A simulation runtime environment with a client server architecture over multiple protocols". In: Proceedings of the 12th International Modelica Conference, Prague, Czech Republic, , 2017. Linköping University Electronic Press, May 15, 2017. DOI: $10.3384 /$ ecp17132653.

[55] A. Mengist et al. "An Open-Source Composite Modeling Editor and Simulation Tool Based on FMI and TLM Co-Simulation". In: Proceedings of the 11th International Modelica Conference. Linköping University Electronic Press, Sept. 2015. DOI: 10.3384/ecp15118181.

[56] David Kleidermacher and Mike Kleidermacher. Embedded Systems Security: Practical Methods for Safe and Secure Software and Systems Development. Elsevier Science, Apr. 25, 2012, pp. 182-187. ISBN: 9780123868879 .

[57] Magnus Eek, Johan Naeser, Nils Paulsson, Robert Hällqvist, Alexander Yngve, and Frank Peterson. D2.5: Integration of the FMI standard: Annex A-Saab. Tech. rep. Saab Aeronautics, Mar. 12, 2018.

[58] Tom Schierz, Martin Arnold, and Christoph Clauss. "Co-simulation with communication step size control in an FMI compatible master algorithm". In: Proceedings of the 9th International MODELICA Conference, September 3-5, 2012, Munich, Germany. Linköping University Electronic Press, Nov. 2012. DOI: 10.3384/ecp12076205.

[59] Martin Benedikt and Anton Hofer. "Guidelines for the Application of a Coupling Method for Non-iterative Co-simulation". In: 2013 8th EUROSIM Congress on Modelling and Simulation. IEEE, Sept. 2013. DOI: 10.1109/EUROSIM. 2013.52.

[60] Torkel Glad and Lennart Ljung. Control Theory-Multivariable and Nonlinear Methods. Taylor and Francis Ltd, 2000. ISBN: 9780748408788.

[61] G. Heinzel, A. Rudiger, and R. Schilling. Spectrum and spectral density estimation by the Discrete Fourier transform (DFT), including a comprehensive list of window functions and some new flat-top windows. Report. Max-Planck-Institut fur Gravitationsphysik, Feb. 15, 2002.

[62] Torkel Glad and Lennart Ljung. Reglerteknik: Grundläggande teori. Studentlitteratur AB, Oct. 1, 2006. ISBN: 9789144022758.

[63] Petter Krus. "Modeling of Mechanical Systems Using Rigid Bodies and Transmission Line Joints". In: Journal of dynamic systems, measurement, and control 121.4 (Dec. 1999), p. 606. DOI: 10.1115/1.2802523. 
[64] D. M. Auslander. "Distributed System Simulation With Bilateral Delay-Line Models". In: Journal of Basic Engineering 90.2 (1968). DOI: https://doi.org/10.1115/1.3605079.

[65] Peter B. Johns and Mark O'Brien. "Use of the transmission-line modelling (t.l.m.) method to solve non-linear lumped networks". In: Radio and Electronic Engineer 50.1-2 (1980). DOI: 10.1049/ree.1980.0006.

[66] Petter Krus, Arne Jansson, Jan-Ove Palmberg, and Kenneth Weddfeldt. "Distributed Simulation of Hydromechanical Systems". In: Third Bath International Fluid Power Workshop. Jan. 1990.

[67] Björn Eriksson, Peter Nordin, and Petter Krus. "Hopsan NG, A C++ Implementation using the TLM Simulation Technique". In: Proceedings of the 51st conference on simulation and modelling. Finland, Oulu, Oct. 2010 .

[68] Mikael Axin, Robert Braun, Alessandro Dell'Amico, and Björn Eriksson. "Next Generation Simulation Software using Transmission Line Elements". In: Fluid Power and Motion Control. Bath, England, UK, 2010 .

[69] Martin Sjölund, Robert Braun, Peter Fritzon, and Petter Krus. "Towards Efficient Distributed Simulation in Modelica using Transmission Line Modeling". In: 3rd International Workshop on Equation-Based Object-Oriented Modeling Languages and Tools. 2010.

[70] Dag Fritzon, Robert Braun, and Jan Hartford. "Composite modelling in 3-D mechanics utilizing Transmission Line Modelling (TLM) and Functional Mock-up Interface (FMI)". In: Modeling, Identification and Control (Mar. 4, 2018). DOI: 10.4173/misc.2018.3.4.

[71] Hans Boden, Ulf Carlsson, Ragnar Glav, HP Wallin, and Mats Åbom. Ljud och Vibrationer. June 1999. ISBN: 91-7170-434-5.

[72] Robert Braun, Adeel Asghar, Adrian Pop, and Dag Fritzson. "An Open-Source Framework for Efficient Co-simulation of Fluid Power Systems". In: The 15th Scandinavian International Conference on Fluid Power. Linköping, Sweden, 2017.

[73] O. Balci and William F. Ormsby. "Well-defined intended uses: an explicit requirement for accreditation of modeling and simulation applications". In: 2000 Winter Simulation Conference Proceedings (Cat. No.00CH37165). IEEE. DOI: 10.1109/WSC.2000.899883.

[74] Charles H. Piersall III and Franklin E Grange. "The Necessity of Intended Use Specification for Successful Modeling and Simulation of a System-of-Systems." In: Crosstalk, the Journal of Defense Software Engineering (2014), pp. 25-28. 
[75] William L. Oberkampf and Christopher J. Roy. Verification and Validation in Scientific Computing. Cambridge University Press, Jan. 13, 2014, pp. 378-381. 790 pp. ISBN: 978-0-521-11360-1.

[76] Standards for Models and Simulations, NASA STD-7009. Washington DC: National Aeronautics and Space Administration, 2008.

[77] Manfred Roza, Jeroen Voogd, and Derek Sebalj. "The Generic Methodology for Verification and Validation to support acceptance of models, simulations and data". In: The Journal of Defense Modeling and Simulation: Applications, Methodology, Technology 10.4 (Oct. 2012), pp. 347-365. DOI: $10.1177 / 1548512912459688$.

[78] Department of Defence Directive Number 5000.59. US Department of Defence, 2007.

[79] Sezer Atamturktur, Matthew C. Egeberg, François M. Hemez, and Garrison N. Stevens. "Defining coverage of an operational domain using a modified nearest-neighbor metric". In: Mechanical Systems and Signal Processing 50-51 (Jan. 2015), pp. 349-361. DOI: https://doi .org/ 10.1016/j.ymssp. 2014.05.040.

[80] Sezer Atamturktur, François Hemez, Cetin Unal, and Brian Williams. "Predictive Maturity of Computer Models Using Functional and Multivariate Output". In: Proceedings of the IMAC-XXVII. 2009.

[81] François Hemez, Sezer Atamturktur, and Cetin Unal. "Defining predictive maturity for validated numerical simulations". In: Computers and Structures 88 88.7-8 (Apr. 2010), pp. 497-505. DOI: 10.1016/j . compstruc. 2010.01.005.

[82] Christopher J. Roy and William L. Oberkampf. "A comprehensive framework for verification, validation, and uncertainty quantification in scientific computing". In: Computer methods in applied mechanics and engineering 200.25-28 (June 2011), pp. 2131-2144. DOI: 10.1016/ j.cma.2011.03.016.

[83] Jennifer Chew and Cindy Sullivan. "Verification, validation, and accreditation in the life cycle of models and simulations". In: 2000 Winter Simulation Conference Proceedings (Cat. No.00CH37165). IEEE, 2000. DOI: $10.1109 /$ WSC . 2000.899879.

[84] Anders Andersson and Sogol Kharrazi. "A Framework for Credibility Assessment of a Powertrain Model in Driving Simulator Studies". In: Proceedings of the 36th FISITA World Automotive Congress. 2016.

[85] Carl-Philip Forss. "Analysis and Visualization of Validation Results". MA thesis. Department of Electrical Engineering, 2015. 
[86] Kevin J. Dowding, John R. Red-Horse, Thomas L. Paez, Ivo M. Babuška, Richard G. Hills, and Raul Tempone. "Validation challenge workshop summary". In: Computer Methods in Applied Mechanics and Engineering 197.29-32 (May 1, 2008), pp. 2381-2384. DOI: 10.1016/j . cma.2007.10.015.

[87] Scott Harmon and Simone Youngblood. "A Proposed Model for Simulation Validation Process Maturity". In: The Journal of Defense Modeling and Simulation: Applications, Methodology, Technology 2.4 (Oct. 2005). Ed. by David C. Gross, pp. 179-190. DOI: $10.1177 / 154851290500200402$.

[88] Scott Harmon and Simone Youngblood. "Evolving the validation process maturity model (VPMM)". In: Proceedings of the SISO European Simulation Interoperability Workshop. Edinburgh, Scotland, June 2008.

[89] Sarbari Gupta. A four-phase approach to independent verification and validation. Ed. by GCN. https://gcn.com/Articles/2018/03/27/independentverification-and-validation.aspx. Mar. 27, 2018. (Visited on 11/11/2019).

[90] J.D. Arthur and R.E. Nance. "Verification and Validation without Independence: a recipe for failure". In: 2000 Winter Simulation Conference Proceedings (Cat. No.00CH37165). IEEE, 2000. DOI: 10.1109/WSC . 2000.899885 .

[91] Software Engineering Standards Commitee of the IEEE Computer Society. IEEE Standard for Software Verification and Validation. IEEE Std 1012-1998. Ed. by IEEE-SA Standards Board. Mar. 9, 1998.

[92] Robert Hällqvist. Digital Twin for Automated Flight Test Evaluation and Model Validation. Ed. by VINNOVA. May 14, 2019.

[93] ITEA 3. Environment for model-based rigorous adaptive co-design and operation of CPS. Nov. 12, 2019. URL: https://itea3.org/project/ embrace.html (visited on 11/12/2019).

[94] Herbert E. Merritt. HYDRAULIC CONTROL SYSTEMS. EGU General Assembly Conference Abstracts. John Wiley and Sons, Inc., Apr. 1967. ISBN: 0471596175 . 


\section{Papers}

The papers associated with this thesis have been removed for copyright reasons. For more details about these see:

http://urn.kb.se/resolve?urn=urn:nbn:se:liu:diva-162810 
"Men Herren will jag lofva, att aldrig för min skull, det sucka skall så tungt uti skogen"

Bernard Elis Malmström 


\section{FACULTY OF SCIENCE AND ENGINEERING}

Linköping Studies in Science and Technology, Licentiate Thesis No. 1866, 2019

Department of Management and Engineering

Linköping University

SE-581 83 Linköping, Sweden

www.liu.se 